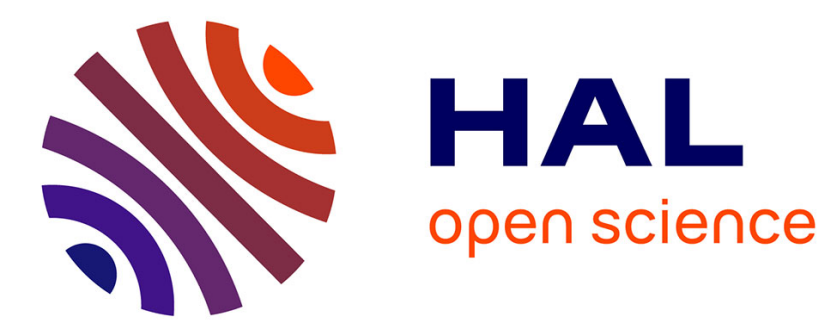

\title{
Fourth order pseudo maximum likelihood methods
} Alberto Holly, Alain Monfort, Michael Rockinger

\section{To cite this version:}

Alberto Holly, Alain Monfort, Michael Rockinger. Fourth order pseudo maximum likelihood methods. Econometrics, 2011, 10.1016/j.jeconom.2011.01.004 • hal-00815562

\section{HAL Id: hal-00815562 https://hal.science/hal-00815562}

Submitted on 19 Apr 2013

HAL is a multi-disciplinary open access archive for the deposit and dissemination of scientific research documents, whether they are published or not. The documents may come from teaching and research institutions in France or abroad, or from public or private research centers.
L'archive ouverte pluridisciplinaire HAL, est destinée au dépôt et à la diffusion de documents scientifiques de niveau recherche, publiés ou non, émanant des établissements d'enseignement et de recherche français ou étrangers, des laboratoires publics ou privés. 


\section{Accepted Manuscript}

Fourth order pseudo maximum likelihood methods

Alberto Holly, Alain Monfort, Michael Rockinger

PII:

S0304-4076(11)00025-X

DOI:

10.1016/j.jeconom.2011.01.004

Reference: ECONOM 3444

To appear in: Journal of Econometrics

Received date: 31 May 2009

Revised date: 22 November 2010

Accepted date: 28 January 2011

Please cite this article as: Holly, A., Monfort, A., Rockinger, M., Fourth order pseudo maximum likelihood methods. Journal of Econometrics (2011), doi:10.1016/j.jeconom.2011.01.004

This is a PDF file of an unedited manuscript that has been accepted for publication. As a service to our customers we are providing this early version of the manuscript. The manuscript will undergo copyediting, typesetting, and review of the resulting proof before it is published in its final form. Please note that during the production process errors may be discovered which could affect the content, and all legal disclaimers that apply to the journal pertain. 


\title{
Fourth Order Pseudo Maximum Likelihood Methods
}

\author{
by Alberto Holly ${ }^{a}$, Alain Monfort ${ }^{b}$, \\ and Michael Rockinger ${ }^{c}$
}

\section{November 2010}

${ }^{a}$ Institute of Health Economics and Management (IEMS), University of Lausanne, Faculty of Business and Economics, Extranef Building, CH-1015 Lausanne, Switzerland. E-mail: alberto.holly@unil.ch.

${ }^{b}$ Corresponding author. CREST, Banque de France, and University of Maastricht. CREST, 15 Boulevard Péri, 92245 Malakoff Cédex, France, E-mail: alain.monfort@ensae.fr.

${ }^{c}$ Swiss Finance Institute, University of Lausanne, and CEPR. University of Lausanne. Faculty of Business and Economics, Extranef Building, CH-1015 Lausanne, Switzerland. E-mail: michael.rockinger@unil.ch.

Keywords: Quartic Exponential Family, Pseudo Maximum Likelihood, Skewness, Kurtosis. JEL classification: C01, C13, C16, C22

Acknowledgement: The third author acknowledges support from the Swiss National Science Foundation through NCCR FINRISK (Financial Valuation and Risk Management). We are grateful to Prof. W. Gautschi for his advice concerning numerical integration and to Prof. G. V. Milovanović for providing a very useful sequence of parameters used for numerical integrations. 


\begin{abstract}
We extend PML theory to account for information on the conditional moments up to order four, but without assuming a parametric model, to avoid a risk of misspecification of the conditional distribution. The key statistical tool is the quartic exponential family, which allows us to generalize the PML2 and QGPML1 methods proposed in Gourieroux, Monfort, and Trognon (1984) to PML4 and QGPML2 methods, respectively. An asymptotic theory is developed. The key numerical tool that we use is the Gauss-Freud integration scheme that solves a computational problem that has previously been raised in several fields. Simulation exercises demonstrate the feasibility and robustness of the methods.
\end{abstract}




\section{INTRODUCTION}

It is well known that the Maximum Likelihood estimator may not only be inefficient but also inconsistent under misspecification, that is when the parametric model providing the likelihood function does not contain the true distribution. The study of the relations between Maximum Likelihood Theory and misspecification has now a long history. Hood and Koopmans (1953) demonstrated that the conditionally Gaussian ML estimator is consistent and asymptotically Gaussian, even if the true distribution is not conditionally Gaussian, as soon as the first two conditional moments are well specified. They coined the label "quasi ML estimator" for this kind of estimator. White (1982) showed that, under misspecification, the ML estimator is in fact a CAN (consistent asymptotically normal) estimator of the pseudo-true value (as defined for instance in Sawa (1978)). Gourieroux, Monfort, and Trognon (1984) characterized the parametric families leading to CAN estimators of the parameters appearing in the first two conditional moments, even if the true distribution does not belong to this parametric family. These families are the linear exponential families (when only the first conditional moment is specified) and the quadratic exponential families (when the first two conditional moments are specified). The estimators thus obtained were called PML1 and PML2 estimators, respectively. Bollerslev and Wooldrige (1992) generalized the properties of the quasi generalized estimator, i.e. the Gaussian PML estimator, to the dynamic case.

The PML theories described above only consider inference on the parameters appearing in the first two conditional moments. More recently, however, many econometric fields are paying greater attention to higher order conditional moments. This is particularly the case in Financial Econometrics and in Health Econometrics. Very often, the approach used to account for higher moments is the ML method based on a choice of a parametric family, which allows for asymmetry and fat tails. A few such examples, occurring in finance are: Generalized Hyperbolic distribution [Eberlein and Keller (1995); Barndorff-Nielsen (1997)], the noncentral Student t distribution [Harvey and Siddique (1999)], and the Skewed-t distribution [Hansen (1994); Jondeau and Rockinger (2003)]. In Health Econometrics, some examples are: The Generalized Gamma distribution proposed by Stacy (1962) and Stacy and Mihram (1965), [Manning, Basu, and 
Mullahy (2005)], and the Pearson Type IV distribution, which may be considered a skewed-t distribution [Holly and Pentsak (2004)]. More generally, examples can be found in various fields, including physics, astronomy, image processing, and in the biomedical sciences [see Genton (2004) and Arellano-Valle and Genton (2005)].

Current ML approaches have two types of drawbacks. First, some families may not be flexible enough to span the whole set of possible skewness $(s)$ and kurtosis $(k)$, namely the domain $k \geq s^{2}+1$. Second, as mentioned above, the risk of misspecification may lead to inconsistent estimators. If we are interested in the first four conditional moments, a natural method is the Generalized Method of Moments (GMM). There is now a large body of studies, however, suggesting that GMM estimators can have poor finite sample properties [see e.g. Tauchen (1986), Andersen and Sorenson (1996), Altonji and Segal (1996), Ziliak (1997), Doran and Schmidt (2006)]. These difficulties led Kitamura and Stutzer (1997), to propose an alternative estimation procedure based on the Kullback-Leibler Information Criterion.

The objective of this paper is to propose another alternative to GMM. It is an extension of the PML method developed by Gourieroux, Monfort, and Trognon (1984), henceforth referred to as GMT. This work extends GMT to a situation where the first four moments (centered or not) are known functions depending on unknown parameters. Specifically, we show that the PML estimator is consistent for any specification of the first four conditional moments, any true conditional distribution of the endogenous variable, and any marginal distribution of the exogenous variables, if and only if the PML is based on a quartic exponential family. We shall refer to this extension as PML4. We also propose an extension of the Quasi-Generalized Pseudo Maximum Likelihood (QGPML) estimator proposed by GMT (1984) based on the quartic exponential family and called QGPML2. Beyond the robustness and nice asymptotic properties of the estimates resulting from the quartic exponential families, two additional features should be noted. First, the quartic exponential family spans the whole set of possible values for the mean and the variance as well as the pairs $(s, k)$, except for a set of measure zero, namely $\{(s, k)$, s.t. $s=0, k>3\}$. This is not necessarily the case for other parametric families of distributions such as those mentioned earlier. Second, we show how the parameters of the quartic exponential family may be obtained from a given set of moments. Thereby, we solve a numerical problem which had been already encountered, both in the econometric literature [Zellner and Highfield (1988), Ormoneit and White (1999)] and other fields [Agmon et al. (1979) or 
Mead and Papanicolaou (1984)], where the exponential family arises as an Entropy Maximizing density and which were considered as difficult. The key issue, from the computational point of view, is the use of the Gauss-Freud quadrature scheme which seems very promising for computing the numerical integrations needed in this framework. It is also important to note that, since the quartic exponential family will be used as a tool providing a convenient set of auxiliary probability distributions generating the whole set of pairs $(s, k)$ except the set of measure zero $\{(s, k), s=0, k>3\}$, it is also possible to exclude the point ( $s=0, k=3$ ) without any practical consequence; this will be done for sake of technical simplicity. Of course, as usual in PML theories, this choice of auxiliary probability distribution does not imply any restriction on the true distribution.

The rest of this paper is organized as follows. Preliminary results are given in Section 2, where some properties of exponential families are briefly reviewed and the notion of a quartic exponential family is defined. This section also contains a brief presentation of the properties of M-Estimators. These preliminary results are then used to derive important properties of the exponential quartic family in Section 3. The PML4 method is defined in Section 4, and the asymptotic properties of the PML4 estimators are derived. In Section 5, we perform a similar analysis as in Section 4 but for the QGPML2 method. In Section 6, we discuss the numerical issues and describe numerical algorithms for implementing of the PML4 and QGPML2 methods. Several Monte-Carlo exercises demonstrating the usefulness of the methods proposed in our paper are presented in Section 7. This section contains a discussion on computational issues linked with the quartic exponential distribution, and it also presents four Monte-Carlo experiments, each of which numerically demonstrates a different property of PML4 or QGPML2. Conclusions are presented in Section 8. Finally, to not interrupt our discussion of the essential ideas of this paper, some proofs and other technical details are presented in the Appendix.

\section{Preliminaries}

\subsection{Exponential Families.}

Let us consider a measure space $(\mathcal{Y}, \mathcal{A}, \nu)$ where $\mathcal{A}$ is a $\sigma$-field and $\nu$ a $\sigma$-finite measure. An exponential family is a family of probability distributions on $(\mathcal{Y}, \mathcal{A})$ which are equivalent to $\nu$ and with pdfs of the form:

$$
\ell(y, \lambda)=\exp \left[\lambda^{\prime} T(y)-\psi(\lambda)\right], \quad \lambda \in \Lambda \subset \mathbb{R}^{p}
$$


where $T(y)$ is a $p$-dimensional vector defined on $\mathcal{Y}$, and $\psi(\lambda)$ is a normalizing constant, equal to the Log-Laplace transform of $\nu^{T}$, equivalent to the image of $\nu$ by $T$.

Such families have many well-known properties. Some of them will be useful in the rest of the paper, and they are summarized below [the proofs can be found for instance in Barndorff-Nielsen (1978), Monfort (1982), or Brown (1986)].

(1) $\Lambda$ can be taken as the convex set where the Laplace transform of $\nu^{T}$ is defined.

(2) For any $\lambda \in \AA$, interior of $\Lambda$, all the moments of the statistic $T$ exist, and in particular, we have:

$$
E_{\lambda}(T)=\frac{\partial}{\partial \lambda} \psi(\lambda), \quad V_{\lambda}(T)=\frac{\partial^{2}}{\partial \lambda \partial \lambda^{\prime}} \psi(\lambda)
$$

which implies:

$$
\frac{\partial E_{\lambda}(T)}{\partial \lambda^{\prime}}=\frac{\partial^{2}}{\partial \lambda \partial \lambda^{\prime}} \psi(\lambda)=V_{\lambda}(T)
$$

(3) The Fisher information matrix $I_{F}(\lambda)$ is equal to $V_{\lambda}(T)=\partial^{2} \psi(\lambda) / \partial \lambda \partial \lambda^{\prime}$.

(4) The model is identifiable if, and only if, $I_{F}(\lambda)$ is invertible for any $\lambda \in \Lambda$.

(5) If the model is identifiable, then the mapping $\lambda \rightarrow E_{\lambda}(T)$ is injective.

\subsection{Quartic Exponential Family.}

We consider the particular case where $\mathcal{Y}=\mathbb{R}, \mathcal{A}=\mathcal{B}_{\mathbb{R}}$ (the Borelian $\sigma$-field of $\mathbb{R}), \nu$ is the Lebesgue measure on $\mathbb{R}$, and $T(y)=\left(y, y^{2}, y^{3}, y^{4}\right)^{\prime}$.

In other words, we consider the pdfs on $\mathbb{R}$ defined by:

$$
\ell(y, \lambda)=\exp \left[\sum_{i=1}^{4} \lambda_{i} y^{i}-\psi(\lambda)\right], \quad \text { with } \lambda=\left(\lambda_{1}, \lambda_{2}, \lambda_{3}, \lambda_{4}\right)^{\prime} .
$$

We will also use the notation:

$$
\ell(y, \lambda)=\exp \left[\lambda_{0}+\sum_{i=1}^{4} \lambda_{i} y^{i}\right], \quad \text { with } \lambda_{0}=-\psi(\lambda) .
$$

This type of density has been extensively used in the entropy literature, e.g. Golan et al. (1996), since it is obtained by maximizing, with respect to $f$, the entropy $-\int_{\mathbb{R}} f(y) \log f(y) d y$, under a set of data moment-consistency constraints $\int_{\mathbb{R}} y^{i} f(y) d y=m_{j}$, for $j=1, \cdots, 4$, where the $m_{j}$ are given, as well as a normalization constraint $\int_{\mathbb{R}} f(y) d y=1$.

The set $\Lambda$ where $\ell(y, \lambda)$ is defined is easily obtained. If $\lambda_{4}<0, \ell(y, \lambda)$ is always integrable. If $\lambda_{4}>0, \ell(y, \lambda)$ is never integrable. Finally, if $\lambda_{4}=0, \ell(y, \lambda)$ is 
integrable if $\lambda_{3}=0$ and $\lambda_{2}<0$ and we get the Gaussian family. In other words, $\Lambda$ is defined by:

$$
\Lambda=\mathbb{R} \times \mathbb{R} \times \mathbb{R} \times \mathbb{R}^{-*}+\mathbb{R} \times \mathbb{R}^{-*} \times\{0\} \times\{0\},
$$

where $\mathbb{R}^{-*}$ is the set of strictly negative numbers.

This family will be called the quartic exponential family and denoted by $\{Q(\lambda)$, $\lambda \in \Lambda\}$.

One should note that the variance-covariance matrix of $T(Y)=\left(Y, Y^{2}, Y^{3}, Y^{4}\right)^{\prime}$ is invertible everywhere, since otherwise there would exist a linear relation between $Y, Y^{2}, Y^{3}, Y^{4}$, i.e. the support of the distribution of $Y$ would be made of at most four points, which is impossible since this distribution is absolutely continuous with respect to the Lebesgue measure. Therefore, using the general properties 3 ) and 4) we see that the model is identifiable. Moreover, using 5) we conclude that the mapping $\lambda \rightarrow\left[m_{i}(\lambda), i=1, \ldots, 4\right]$, where $m_{i}(\lambda)=E_{\lambda}\left(Y^{i}\right)$, is injective.

Denoting by $s(\lambda)$ and $k(\lambda)$ the skewness and kurtosis $\left[s(\lambda)=E_{\lambda}[Y-E(Y)]^{3} /\right.$ $\left.\left[V_{\lambda}(Y)\right]^{3 / 2}, k(\lambda)=E_{\lambda}[Y-E(Y)]^{4} /\left[V_{\lambda}(Y)\right]^{2}\right]$, respectively, it is also clear that the mapping:

$$
\lambda \rightarrow\left[m_{1}(\lambda), m_{2}(\lambda), s(\lambda), k(\lambda)\right]
$$

is injective. The same is true for the mapping

$$
\lambda \rightarrow\left[m(\lambda), \sigma^{2}(\lambda), s(\lambda), k(\lambda)\right]
$$

where $m(\lambda)=m_{1}(\lambda)$, and $\sigma^{2}(\lambda)=m_{2}(\lambda)-m_{1}^{2}(\lambda)$.

It is important to check whether the previous mapping is also surjective, which is to say that it can reach any admissible value of $\left(m_{1}, m_{2}, s, k\right)$. It is well known that the set $\mathcal{D}$ of admissible values of $\left(m, \sigma^{2}, s, k\right)$ is defined by:

$$
m \in \mathbb{R}, \quad \sigma^{2} \geq 0, \quad s \in \mathbb{R}, \quad k \geq s^{2}+1 .
$$

The latter inequality is obtained, for instance, by noting that the variance-covariance matrix of $\left(Y, Y^{2}\right)$ where $E(Y)=0, V(Y)=1$, is given by

$$
\left(\begin{array}{cc}
1 & s \\
s & k-1
\end{array}\right) \text {, }
$$

and therefore that $k-1-s^{2} \geq 0$. Moreover, the boundary $k=s^{2}+1$ is reached if $Y$ and $Y^{2}$ are linked linearly, that is, if the support is made of at most two points. Therefore, this boundary clearly cannot be reached by the quartic family, and the boundary point $\sigma^{2}=0$ cannot be reached either (for the same reason). 
Therefore, the natural question is now the following: is the range of the mapping $\lambda \rightarrow\left[m(\lambda), \sigma^{2}(\lambda), s(\lambda), k(\lambda)\right]$ defined by:

$$
D=\left\{m \in \mathbb{R}, \quad \sigma^{2}>0, \quad s \in \mathbb{R}, \quad k>s^{2}+1\right\} ?
$$

The answer is no, but it can be shown [see Junk (2000)] that the range is almost equal to this set, in the sense that all the admissible values of $\left(m, \sigma^{2}, s, k\right)$ can be reached except for those corresponding to the set of measure zero, defined by $s=0, k>3$.

Moreover, if we exclude the case $\lambda_{4}=0$ (and therefore $\lambda_{3}=0$ ), in other words, if we restrict the $Q(\lambda)$ family to the case where $\lambda_{4}<0$, then the only probability distributions excluded are the normal distributions (corresponding to $s=0$ and $k=3$ ) and, therefore, the range of the $m$ reached by this restricted family is $D$ except the points corresponding to $s=0$, and $k \geq 3$. We will denote by $\Lambda^{*}$ the set $\mathbb{R} \times \mathbb{R} \times \mathbb{R} \times \mathbb{R}^{-*}$, and by $D^{*}$ the set

$$
D^{*}=D-\left\{\left(m, \sigma^{2}, s, k\right), s=0, k \geq 3\right\} .
$$

There is a one to one relationship between $\Lambda^{*}$ and $D^{*}$, and, moreover, all the points of $D$ not belonging to $D^{*}$ can be approached as closely as wished by a distribution of $\left\{Q(\lambda), \lambda \in \Lambda^{*}\right\}$. In the sequel, we will take the quartic exponential family $\left\{Q(\lambda), \lambda \in \Lambda^{*}\right\}$ as the auxiliary family on which the semi-parametric estimator of the parameters of interest will be based. ${ }^{1}$

2.3. M-estimators and Quasi-Generalized M-estimators. Let us consider an endogenous variable $Y_{i}$ and a vector of exogenous variables $X_{i}$. For simplicity, we assume that $\left(Y_{i}, X_{i}\right)$ for $i=1, \cdots, n$ are i.i.d. Standard extensions can be found in Gallant (1987), Holly (1993), or White (1994). To each possible conditional distribution of the $Y_{i}^{\prime}$ 's given the $X_{i}$ 's, we associate a parameter $\theta \in \Theta \subset \mathbb{R}^{K}$. In particular, the value of the parameter corresponding to the true conditional distribution of the $Y_{i}$ 's given the $X_{i}$ 's is called the true value of the parameter, and it is denoted by $\theta_{0}$. The true distribution of the sequence $\left(Y_{i}, X_{i}, i \in \mathbb{N}\right)$ is denoted by $P_{0}$. Throughout this paper, we adopt the notation corresponding to a conditional static model, but the results could be extended to a stationary conditional dynamic model by replacing $Y_{i}$ by $Y_{t}$ and $X_{i}$ by $\left(Y_{t-1}, \ldots, Y_{1}, X_{t}, \ldots, X_{1}\right)$.

\footnotetext{
${ }^{1}$ Note that in particular the moments generated by standard exponential families like the binomial, gamma, and Poisson are reached, since their skewness is non zero.
} 
An M-estimator of $\theta_{0}$ is an estimator $\hat{\theta}_{n}$ obtained by maximizing, with respect to $\theta$, an objective function of the form:

$$
\sum_{i=1}^{n} \varphi\left(Y_{i}, X_{i}, \theta\right)
$$

Under standard regularity conditions [see e.g. Chamberlain (1987), Newey (1990), White (1994), Gourieroux and Monfort (1995a)], it can be shown that $\hat{\theta}_{n}$ is a consistent estimator of $\theta_{0}$, for any $\theta_{0}$, if the limit function

$$
\varphi_{\infty}\left(\theta, P_{0}\right)=P_{0} \lim \left[\frac{1}{n} \sum_{i=1}^{n} \varphi\left(Y_{i}, X_{i}, \theta\right)\right]
$$

has a unique maximum at $\theta=\theta_{0}$. Moreover, the limit function can be written:

$$
\varphi_{\infty}\left(\theta, P_{0}\right)=E_{X} E_{0} \varphi(Y, X, \theta)
$$

where $E_{0}$ is the conditional expectation operator associated to the true conditional distribution of $Y_{i}$, given that $X_{i}=x$ (independent of $i$ ) and $E_{X}$ is the expectation with respect to the distribution $P_{X}$, of any $X_{i}$.

Let us now consider two subvectors $\theta^{*}$ and $\theta^{* *}$ of $\theta$. These subvectors are not necessarily disjoint, and in particular, we can have $\theta^{*}=\theta^{* *}=\theta$.

We assume that $\theta_{0}^{*}$, the true value of $\theta^{*}$, can be consistently estimated by $\hat{\theta}_{n}^{*}$ defined by:

$$
\hat{\theta}_{n}^{*}=\underset{\theta^{*}}{\operatorname{Argmax}} \sum_{i=1}^{n} \varphi\left[Y_{i}, X_{i}, \theta^{*}, a\left(X_{i}, \hat{\theta}_{n}^{* *}\right)\right],
$$

where $a$ is some function, and $\hat{\theta}_{n}^{* *}$ is a consistent estimator of $\theta_{0}^{* *}$, the true value of $\theta^{* *}$. In other words, $\theta_{0}^{*}$ gives the unique maximum in $\theta^{*}$ of:

$$
\begin{aligned}
& P_{0} \lim \left[\frac{1}{n} \sum_{i=1}^{n} \varphi\left[Y_{i}, X_{i}, \theta^{*}, a\left(X_{i}, \hat{\theta}_{n}^{* *}\right)\right]\right] \\
& =E_{X} E_{0} \varphi\left[Y, X, \theta^{*}, a\left(X, \theta_{0}^{* *}\right)\right] .
\end{aligned}
$$

Such an estimator is called a Quasi-Generalized M-estimator of $\theta_{0}^{*}$ (QGM estimator). The corresponding unfeasible M-estimator $\hat{\theta}_{0 n}^{*}$ is defined by:

$$
\hat{\theta}_{0 n}^{*}=\underset{\theta^{*}}{\operatorname{Argmax}} \sum_{i=1}^{n} \varphi\left[Y_{i}, X_{i}, \theta^{*}, a\left(X_{i}, \theta_{0}^{* *}\right)\right],
$$

and is also consistent.

As far as the asymptotic normality of the M and QGM estimators is concerned, we have the following properties. 
The asymptotic distribution of $\sqrt{n}\left(\hat{\theta}_{n}-\theta_{0}\right)$ is $\mathcal{N}\left[0, J^{-1}\left(\theta_{0}\right) I\left(\theta_{0}\right) J^{-1}\left(\theta_{0}\right)\right]$ where

$$
\begin{aligned}
& J\left(\theta_{0}\right)=-E_{X} E_{0}\left[\frac{\partial^{2} \varphi\left(Y, X, \theta_{0}\right)}{\partial \theta \partial \theta^{\prime}}\right], \\
& I\left(\theta_{0}\right)=E_{X} E_{0}\left[\frac{\partial \varphi\left(Y, X, \theta_{0}\right)}{\partial \theta} \frac{\partial \varphi\left(Y, X, \theta_{0}\right)}{\partial \theta^{\prime}}\right] .
\end{aligned}
$$

A nice property of the QGM-estimator $\hat{\theta}_{n}^{*}$ of $\theta_{0}^{*}$ is the following. If

$$
E_{0}\left[\frac{\partial^{2} \varphi\left(Y, X, \theta_{0}^{*}, a\left(X, \theta_{0}^{* *}\right)\right)}{\partial \theta^{*} \partial a^{\prime}} \mid X\right]=0
$$

then $\sqrt{n}\left(\hat{\theta}_{n}^{*}-\theta_{0}^{*}\right)$ has the same asymptotic distribution as $\sqrt{n}\left(\hat{\theta}_{0 n}^{*}-\theta_{0}^{*}\right)$. Namely, $\mathcal{N}\left[0, \tilde{J}^{-1}\left(\theta_{0}\right) \tilde{I}\left(\theta_{0}\right) \tilde{J}^{-1}\left(\theta_{0}\right)\right]$, where $\tilde{J}\left(\theta_{0}\right)$ and $\tilde{I}\left(\theta_{0}\right)$ are of the same form as $(2.9)$ and $(2.10), \theta$ being replaced by $\theta^{*}$ and $\varphi(Y, X, \theta)$ by $\varphi\left(Y, X, \theta^{*}, a\left(X, \theta_{0}^{* *}\right)\right)$.

\section{Properties of the Exponential Quartic Family}

Let us denote by:

$$
\ell(y, \lambda)=\exp \left(\lambda_{0}+\lambda_{1} y+\lambda_{2} y^{2}+\lambda_{3} y^{3}+\lambda_{4} y^{4}\right)
$$

the pdf of the exponential family, and where $\lambda=\left(\lambda_{1}, \lambda_{2}, \lambda_{3}, \lambda_{4}\right)^{\prime}$, and $\lambda \in \Lambda^{*}=$ $\mathbb{R}^{3} \times \mathbb{R}^{-*}$. We know that there is a one to one relationship between $\Lambda^{*}$ and $D^{*}$ (see Section 2.2). Let us denote by $M$ the range of $m=\left(m_{1}, m_{2}, m_{3}, m_{4}\right)^{\prime}$, where $m_{i}=E\left(Y^{i}\right)$ corresponding to $D^{*}$. The mapping $m(\lambda)$ from $\Lambda^{*}$ to $M$ is bijective, and we denote by $\lambda(m)$ the inverse function and $\lambda_{0}(m)=-\psi[\lambda(m)]$.

Proposition 1. We have:

$$
\frac{\partial \lambda_{0}(m)}{\partial m}+\frac{\partial \lambda^{\prime}(m)}{\partial m} m=0
$$

Proof. We have:

$$
\begin{gathered}
\log \ell[y, \lambda(m)]=\lambda_{0}(m)+\sum_{j=1}^{4} \lambda_{j}(m) y^{j}, \\
\frac{\partial \log \ell[y, \lambda(m)]}{\partial m}=\frac{\partial \lambda_{0}(m)}{\partial m}+\sum_{j=1}^{4} \frac{\partial \lambda_{j}(m)}{\partial m} y^{j} .
\end{gathered}
$$

The result follows by taking the expectation and using the fact that the score vector is of zero mean.

Corollary 1. We have:

$$
\frac{\partial^{2} \lambda_{0}(m)}{\partial m \partial m^{\prime}}+\sum_{j=1}^{4} \frac{\partial^{2} \lambda_{j}(m)}{\partial m \partial m^{\prime}} m_{j}+\frac{\partial \lambda^{\prime}(m)}{\partial m}=0 .
$$

Proof. The proof is straightforward by differentiating the identity (3.1) of Proposition 1 once more. 
Let us denote by $\Sigma$ the variance-covariance matrix of $T(Y)=\left(Y, Y^{2}, Y^{3}, Y^{4}\right)^{\prime}$ which is positive definite (since the support of $Y$ is not reduced to point masses).

Proposition 2. We have $\partial m / \partial \lambda^{\prime}=\Sigma$, and therefore, $\partial \lambda / \partial m^{\prime}=\Sigma^{-1}$.

Proof. This is a direct consequence of the general property 2) of Subsection 2.1.

Proposition 3. For any pair $m, m_{0} \in M$, we have:

$$
\lambda_{0}(m)+\lambda^{\prime}(m) m_{0} \leq \lambda_{0}\left(m_{0}\right)+\lambda^{\prime}\left(m_{0}\right) m_{0},
$$

and the equality holds if and only if $m=m_{0}$.

Proof. From Kullback's inequality, we know that:

$$
E_{m_{0}}[\log \ell[y, \lambda(m)]] \leq E_{m_{0}}\left[\log \ell\left[y, \lambda\left(m_{0}\right)\right]\right],
$$

or

$$
E_{m_{0}}\left[\lambda_{0}(m)+\lambda^{\prime}(m) T(Y)\right] \leq E_{m_{0}}\left[\lambda_{0}\left(m_{0}\right)+\lambda^{\prime}\left(m_{0}\right) T(Y)\right]
$$

or

$$
\lambda_{0}(m)+\lambda^{\prime}(m) m_{0} \leq \lambda_{0}\left(m_{0}\right)+\lambda^{\prime}\left(m_{0}\right) m_{0},
$$

and the inequality holds.

Moreover, $m_{0}$ is the unique maximum of $\lambda_{0}(m)+\lambda^{\prime}(m) m_{0}$ for the following reasons. When equality holds in Kullback's inequality, we have, because of the strict concavity of the $\log$ function, $l[y, \lambda(m)]=l\left[y, \lambda\left(m_{0}\right)\right]$ almost everywhere, therefore $\lambda(m)=\lambda\left(m_{0}\right)$ because the quartic family is identifiable (see section 2.2) and, finally, $m=m_{0}$ since the mapping between $\lambda$ and $m$ is one to one.

\section{PML 4 Method}

\subsection{Definition.}

We adopt a semi-parametric approach based on the specification of the conditional moments up to fourth order. It is obviously equivalent to specifying $\left(m_{1}, m_{2}, m_{3}, m_{4}\right)$ or $\left(m_{1}, \sigma^{2}, s, k\right)$. Moreover, to satisfy the inequality $k>s^{2}+1$, it could be convenient to specify $\left(m_{1}, \sigma^{2}, s, k^{*}\right)$, where $k^{*}=k-s^{2}-1$, which could be called the over-kurtosis, since $\left(s, k^{*}\right)$ is only constrained to belong to $\mathbb{R}^{+} \times \mathbb{R}^{+}$. We consider the latter parametrization, but the results could be adapted to other parametrizations in a straightforward manner.

We therefore specify the following functions: $m\left(x_{i}, \theta_{1}\right), \sigma^{2}\left(x_{i}, \theta_{2}\right), s\left(x_{i}, \theta_{3}\right)$, and $k^{*}\left(x_{i}, \theta_{4}\right)$. Note that $\theta_{1}, \theta_{2}, \theta_{3}$, and $\theta_{4}$ may have some components in common, and we denote by $\theta$ the union of $\theta_{1}, \theta_{2}, \theta_{3}$, and $\theta_{4}$ without repetition (in particular, we could have $\theta_{1}=\theta_{2}=\theta_{3}=\theta_{4}=\theta$ ). We denote by $\Theta$ the range of $\theta$. 
For a given $x_{i}$ and $\theta$, we can compute the coefficients $\lambda_{0}, \lambda_{1}, \lambda_{2}, \lambda_{3}, \lambda_{4}$ of the quartic exponential distribution having the same mean, variance, skewness and kurtosis. As mentioned in Section 2, this can always be done unless the skewness is zero and the kurtosis larger than 3 , but even then these values can be closely approached. Let us denote these coefficients by $\lambda_{j}\left(x_{i}, \theta\right), j=0, \ldots, 4$.

Definition 1. The fourth order Pseudo Maximum Likelihood estimator of $\theta_{0}$, called PML4 and denoted by $\hat{\theta}_{n}$ is defined by:

$$
\hat{\theta}_{n}=\underset{\theta \in \Theta}{\operatorname{Argmax}} \sum_{i=1}^{n} \sum_{j=0}^{4} \lambda_{j}\left(x_{i}, \theta\right) y_{i}^{j} .
$$

Condition 1. We assume that the semiparametric model is identifiable, i.e. that:

(1) if $m_{1}\left(x_{i}, \theta_{1}\right)=m_{1}\left(x_{i}, \bar{\theta}_{1}\right), \sigma^{2}\left(x_{i}, \theta_{2}\right)=\sigma^{2}\left(x_{i}, \bar{\theta}_{2}\right), s\left(x_{i}, \theta_{3}\right)=s\left(x_{i}, \bar{\theta}_{3}\right)$, $k^{*}\left(x_{i}, \theta_{4}\right)=k^{*}\left(x_{i}, \bar{\theta}_{4}\right)\left(P_{X}\right.$ almost surely), then we have $\theta=\bar{\theta}$.

Note that Condition 1 is equivalent to

(2) $\lambda_{j}\left(x_{i}, \theta\right)=\lambda_{j}\left(x_{i}, \bar{\theta}\right), j=0, \ldots, 4\left(P_{X}\right.$ almost surely) implies $\theta=\bar{\theta}$.

It is important to stress that the exponential quartic family is a tool providing estimation procedures but that we do not assume that the true (conditional) p.d.f. belongs to this family.

\subsection{Asymptotic Properties.}

Proposition 4. Under standard regularity conditions, if the semi-parametric model is identifiable, the PML4 estimator $\hat{\theta}_{n}$ is consistent.

Proof. From the properties of the M estimators mentioned in Subsection 2.3, we have to prove that the limit function (2.4) $\varphi_{\infty}\left(\theta, P_{0}\right)=E_{X} E_{0} \varphi(Y, X, \theta)$ has a unique maximum at $\theta_{0}$. Here we have:

$$
\begin{aligned}
\varphi_{\infty}\left(\theta, P_{0}\right) & =E_{X} E_{0}\left[\sum_{j=0}^{4} \lambda_{j}(X, \theta) Y^{j}\right], \\
& =E_{X}\left[\lambda_{0}(X, \theta)+\sum_{j=1}^{4} \lambda_{j}(X, \theta) m_{j 0}\right] .
\end{aligned}
$$

Using Proposition 3, we know that

$$
\varphi_{\infty}\left(\theta, P_{0}\right) \leq \varphi_{\infty}\left(\theta_{0}, P_{0}\right)
$$

and that $\varphi_{\infty}\left(\theta, P_{0}\right)=\varphi_{\infty}\left(\theta_{0}, P_{0}\right)$ if and only if $\lambda_{j}(X, \theta)=\lambda_{j}\left(X, \theta_{0}\right), j=0, \ldots, 4$, $P_{X}$ almost surely in $X$, and, therefore, using the identification assumption, if and only if $\theta=\theta_{0}$. The result follows. 
Proposition 5. Under standard regularity conditions, if the semi-parametric model is identifiable, $\sqrt{n}\left(\hat{\theta}_{n}-\theta_{0}\right)$ is asymptotically distributed as

$$
\mathcal{N}\left[0, J^{-1}\left(\theta_{0}\right) I\left(\theta_{0}\right) J^{-1}\left(\theta_{0}\right)\right],
$$

where

$$
\begin{aligned}
& J\left(\theta_{0}\right)=E_{X}\left[\frac{\partial m^{\prime}\left(X, \theta_{0}\right)}{\partial \theta} \Sigma^{-1}\left(X, \theta_{0}\right) \frac{\partial m\left(X, \theta_{0}\right)}{\partial \theta^{\prime}}\right], \\
& I\left(\theta_{0}\right)=E_{X}\left[\frac{\partial m^{\prime}\left(X, \theta_{0}\right)}{\partial \theta} \Sigma^{-1}\left(X, \theta_{0}\right) \Omega(X) \Sigma^{-1}\left(X, \theta_{0}\right) \frac{\partial m\left(X, \theta_{0}\right)}{\partial \theta^{\prime}}\right],
\end{aligned}
$$

where $\Sigma\left(X, \theta_{0}\right)$ is the conditional variance-covariance matrix of $T(Y)=\left(Y, Y^{2}\right.$, $\left.Y^{3}, Y^{4}\right)^{\prime}$ given $X$ in the quartic conditional distribution associated with $\lambda_{j}\left(X, \theta_{0}\right)$, $j=0, \ldots, 4$, and where $\Omega(X)$ is the true conditional variance-covariance matrix of $T$ given $X$.

Proof. See Appendix A.

Formulas giving $J\left(\theta_{0}\right)$ and $I\left(\theta_{0}\right)$ contain the Jacobian matrices $\partial m\left(X, \theta_{0}\right) /$ $\partial \theta^{\prime}$. If the parametrization used is not $m=\left(m_{1}, m_{2}, m_{3}, m_{4}\right)^{\prime}$ but instead $\mu=$ $\left(m_{1}, \sigma^{2}, s, k^{*}\right)^{\prime}$, we must compute $\partial m\left(X, \theta_{0}\right) / \partial \theta^{\prime}$ as a function of $\mu=\left[m_{1}\left(X, \theta_{1}\right)\right.$, $\sigma^{2}\left(X, \theta_{2}\right), s\left(X, \theta_{3}\right)$, and $\left.k^{*}\left(X, \theta_{4}\right)\right]^{\prime}$, and we get:

$$
\frac{\partial m\left(X, \theta_{0}\right)}{\partial \theta^{\prime}}=\frac{\partial m}{\partial \mu^{\prime}} \frac{\partial \mu\left(X, \theta_{0}\right)}{\partial \theta^{\prime}} .
$$

Therefore:

Corollary 2. We have,

$$
\begin{aligned}
J\left(\theta_{0}\right) & =E_{X}\left[\frac{\partial \mu^{\prime}\left(X, \theta_{0}\right)}{\partial \theta} \frac{\partial m^{\prime}}{\partial \mu} \Sigma^{-1}\left(X, \theta_{0}\right) \frac{\partial m}{\partial \mu^{\prime}} \frac{\partial \mu\left(X, \theta_{0}\right)}{\partial \theta^{\prime}}\right], \\
I\left(\theta_{0}\right) & =E_{X}\left[\frac{\partial \mu^{\prime}\left(X, \theta_{0}\right)}{\partial \theta} \frac{\partial m^{\prime}}{\partial \mu} \Sigma^{-1}\left(X, \theta_{0}\right) \Omega(X)\right. \\
& \left.\times \Sigma^{-1}\left(X, \theta_{0}\right) \frac{\partial m}{\partial \mu^{\prime}} \frac{\partial \mu\left(X, \theta_{0}\right)}{\partial \theta^{\prime}}\right] .
\end{aligned}
$$

Propositions 4 and 5 show that the PML4 method based on the quartic exponential family provides consistent and asymptotically normal estimators of the parameters specifying the conditional moments of order one to four. It is also important to note that the unique family with these properties is the generalized quartic family:

$$
\exp \left[\lambda_{0}(m)+\sum_{i=1}^{4} \lambda_{i}(m) y^{i}+a(y)\right],
$$

where $m=\left(m_{1}, m_{2}, m_{3}, m_{4}\right)$. 
Proposition 6. Let $f(y, m)$ be a family of pdfs on $\mathbb{R}$ indexed by their moments $m=\left(m_{1}, m_{2}, m_{3}, m_{4}\right)^{\prime}$. If the PML method based on the maximization of

$$
\sum_{i=1}^{n} \log f\left[y_{i}, m_{1}\left(x_{i}, \theta_{1}\right), m_{2}\left(x_{i}, \theta_{2}\right), m_{3}\left(x_{i}, \theta_{3}\right), m_{4}\left(x_{i}, \theta_{4}\right)\right]
$$

is consistent for any specification of the conditional moments, any true conditional distribution satisfying the moment specification for some value $\theta_{0}$ of $\theta=\left(\theta_{1}, \ldots, \theta_{4}\right)^{\prime}$, and any distribution $P_{X}$ of $X$, then $f(y, m)$ is of the type:

$$
f(y, m)=\exp \left[\lambda_{0}(m)+\sum_{i=1}^{4} \lambda_{i}(m) y^{i}+a(y)\right] .
$$

Proof. Under the assumptions of Proposition 6, we must have, in particular, the consistency property in a model without exogenous variables. Furthermore, this model must possess the parametrization $\theta_{i}=E\left(Y^{i}\right), i=1, \ldots, 4$, where $\theta=$ $\left(\theta_{1}, \theta_{2}, \theta_{3}, \theta_{4}\right)^{\prime}$ belongs to the interior of the domain defined by:

$$
\left|\begin{array}{cc}
1 & \theta_{1} \\
\theta_{1} & \theta_{2}
\end{array}\right| \geq 0 ; \quad\left|\begin{array}{ccc}
1 & \theta_{1} & \theta_{2} \\
\theta_{1} & \theta_{2} & \theta_{3} \\
\theta_{2} & \theta_{3} & \theta_{4}
\end{array}\right| \geq 0
$$

In other words, if for all $\theta_{i}(i=1, \ldots, 4)$ we have $E\left(Y^{i}-\theta_{i}\right)=0$, then we must have:

$$
E\left[\frac{\partial \log f(Y, \theta)}{\partial \theta}\right]=0
$$

Using a version of the Farkas Lemma [see Lemma 8.1 in Gourieroux-Monfort (1995a), p. 252], we conclude that:

$$
\frac{\partial \log f(y, \theta)}{\partial \theta}=\sum_{i=1}^{4} \lambda_{i}(\theta)\left(y^{i}-\theta_{i}\right)
$$

Integrating the latter equation gives the result.

Note that the generalized quartic family can be seen as a quartic family with respect to the modified measure $d \nu^{*}(y)=\exp (a(y)) d \nu(y), \nu$ being the Lebesgue measure on $\mathbb{R}$.

\section{QGPML2 Method}

\subsection{Alternative Parametrization.}

We have seen that the quartic exponential family can be equivalently parametrized by $\lambda=\left(\lambda_{1}, \lambda_{2}, \lambda_{3}, \lambda_{4}\right)^{\prime}$, by $m=\left(m_{1}, m_{2}, m_{3}, m_{4}\right)^{\prime}$, or by $\mu=\left(m_{1}, \sigma^{2}, s, k^{*}\right)^{\prime}$. There is a fourth parametrization that will be of great interest, namely $\left(m_{1}, m_{2}\right.$, $\left.\lambda_{3}, \lambda_{4}\right)^{\prime}$ or equivalently $\nu=\left(m_{1}, \sigma^{2}, \lambda_{3}, \lambda_{4}\right)^{\prime}$. First, we have to show that this is indeed a genuine parametrization. 
Proposition 7 . There is a one to one relationship between $\left(\lambda_{1}, \lambda_{2}, \lambda_{3}, \lambda_{4}\right)$ and $\left(m_{1}, m_{2}, \lambda_{3}, \lambda_{4}\right)$.

Proof. We have to prove that for any $\left(\lambda_{3}, \lambda_{4}\right)$ the relationship

$$
\left(\lambda_{1}, \lambda_{2}\right) \rightarrow\left[m_{1}\left(\lambda_{1}, \lambda_{2}, \lambda_{3}, \lambda_{4}\right), m_{2}\left(\lambda_{1}, \lambda_{2}, \lambda_{3}, \lambda_{4}\right)\right]
$$

is one to one. We have seen that the Jacobian matrix $\partial m / \partial \lambda^{\prime}=\Sigma$ is symmetric and positive definite $\forall m$, so the same is true for the upper $(2 \times 2)$ block-diagonal submatrix. Moreover, for any given $\left(\lambda_{3}, \lambda_{4}\right)$ fixed, the section of $\Lambda^{*}$ is convex, and therefore, using Theorem 6 in Gale-Nikaido (1965), we obtain the required result.

The previous result means that, starting from the quartic family $Q\left(\Lambda^{*}\right)$, we can reparameterize it as $Q\left(m_{1}, \sigma^{2}, \lambda_{3}, \lambda_{4}\right)$, and therefore, fixing $\left(\lambda_{3}, \lambda_{4}\right)$ at any admissible value $\left(\lambda_{3}^{0}, \lambda_{4}^{0}\right)$, we get a quadratic exponential family $Q\left(m_{1}, \sigma^{2}, \lambda_{3}^{0}, \lambda_{4}^{0}\right)$ in the sense of GMT (1984). We denote by $\lambda_{1}^{*}\left(m_{1}, \sigma^{2}, \lambda_{3}, \lambda_{4}\right), \lambda_{2}^{*}\left(m_{1}, \sigma^{2}, \lambda_{3}, \lambda_{4}\right)$ and $\lambda_{0}^{*}\left(m_{1}, \sigma^{2}, \lambda_{3}, \lambda_{4}\right)$ the functions giving $\lambda_{1}, \lambda_{2}, \lambda_{0}$ in terms of $m_{1}, \sigma^{2}, \lambda_{3}, \lambda_{4}$.

\subsection{QGPML2 Method.}

We assume that the conditional mean and variance are specified as $m_{1}\left(X_{i}, \theta_{1}\right)$ and $\sigma^{2}\left(X_{i}, \theta_{2}\right)$, and the conditional skewness and over-kurtosis are specified as $s\left(X_{i}, \theta_{3}\right)$ and $k^{*}\left(X_{i}, \theta_{4}\right)$.

We can first estimate $\left(\theta_{1}, \theta_{2}\right)$ by the PML2 method based on the Gaussian family, i.e. by solving the problem:

$$
\left(\tilde{\theta}_{1 n}, \tilde{\theta}_{2 n}\right)=\underset{\theta_{1}, \theta_{2}}{\operatorname{Argmin}} \sum_{i=1}^{n} \log \sigma^{2}\left(X_{i}, \theta_{2}\right)+\frac{\left[Y_{i}-m_{1}\left(X_{i}, \theta_{1}\right)\right]^{2}}{\sigma^{2}\left(X_{i}, \theta_{2}\right)} .
$$

Next, we compute

$$
\hat{u}_{i}=\frac{Y_{i}-m_{1}\left(X_{i}, \tilde{\theta}_{1 n}\right)}{\sigma\left(X_{i}, \tilde{\theta}_{2 n}\right)}
$$

and obtain consistent estimators of $\tilde{\theta}_{3 n}$ and $\tilde{\theta}_{4 n}$ of $\theta_{3}$ and $\theta_{4}$ from the nonlinear regressions of $\hat{u}_{i}^{3}$ on $s\left(X_{i}, \theta_{3}\right)$ and $\hat{u}_{i}^{4}-s\left(X_{i}, \tilde{\theta}_{3 n}\right)^{2}-1$ on $k^{*}\left(X_{i}, \theta_{4}\right)$. Explicitly, this corresponds to obtaining the pair $\left(\tilde{\theta}_{3 n}, \tilde{\theta}_{4 n}\right)$, verifying:

$$
\begin{aligned}
& \tilde{\theta}_{3 n}=\underset{\theta_{3}}{\operatorname{argmin}} \sum_{i=1}^{N}\left(\hat{u}_{i}^{3}-s\left(X_{i}, \theta_{3}\right)\right)^{2}, \\
& \tilde{\theta}_{4 n}=\underset{\theta_{4}}{\operatorname{argmin}} \sum_{i=1}^{N}\left(\hat{u}_{i}^{4}-s\left(X_{i}, \tilde{\theta}_{3 n}\right)^{2}-1-k^{*}\left(X_{i}, \theta_{4}\right)\right)^{2} .
\end{aligned}
$$


Then, noting $\tilde{\theta}_{n}=\left(\tilde{\theta}_{1 n}, \tilde{\theta}_{2 n}, \tilde{\theta}_{3 n}, \tilde{\theta}_{4 n}\right)^{\prime}$, we define:

$$
\begin{gathered}
\tilde{m}_{1 i}=m_{1}\left(x_{i}, \tilde{\theta}_{1 n}\right) ; \quad \tilde{\sigma}_{i}^{2}=\sigma^{2}\left(x_{i}, \tilde{\theta}_{2 n}\right) ; \\
\tilde{s}_{i}=s\left(x_{i}, \tilde{\theta}_{3 n}\right) ; \quad \tilde{k}_{i}^{*}=k^{*}\left(x_{i}, \tilde{\theta}_{4 n}\right) ; \\
\tilde{\lambda}_{j i}=\lambda_{j}\left(\tilde{m}_{1 i}, \tilde{\sigma}_{i}^{2}, \tilde{s}_{i}, \tilde{k}_{i}^{*}\right), \quad j=3,4 .
\end{gathered}
$$

Definition 2. The Quasi Generalized PML2 (QGPML2) estimator $\left(\hat{\theta}_{1 n}, \hat{\theta}_{2 n}\right)$ of $\left(\theta_{01}, \theta_{02}\right)$ is defined by maximizing with respect to $\left(\theta_{1}, \theta_{2}\right)$ :

$$
\begin{aligned}
L_{n}^{(2)}\left(\theta_{1}, \theta_{2}\right) & =\sum_{i=1}^{n}\left\{\lambda_{0}^{*}\left[m_{1}\left(x_{i}, \theta_{1}\right), \sigma^{2}\left(x_{i}, \theta_{2}\right), \tilde{\lambda}_{3 i}, \tilde{\lambda}_{4 i}\right]\right. \\
& +\lambda_{1}^{*}\left[m_{1}\left(x_{i}, \theta_{1}\right), \sigma^{2}\left(x_{i}, \theta_{2}\right), \tilde{\lambda}_{3 i}, \tilde{\lambda}_{4 i}\right] y_{i} \\
& \left.+\lambda_{2}^{*}\left[m_{1}\left(x_{i}, \theta_{1}\right), \sigma^{2}\left(x_{i}, \theta_{2}\right), \tilde{\lambda}_{3 i}, \tilde{\lambda}_{4 i}\right] y_{i}^{2}\right\}
\end{aligned}
$$

Note that, using the parametrization $\left(m_{1}, \sigma^{2}, \lambda_{3}, \lambda_{4}\right)$ the quartic family of pdf can be written:

$$
\begin{aligned}
& f\left(y_{i} \mid m_{1}, \sigma^{2}, \lambda_{3}, \lambda_{4}\right)=\exp \left[\lambda_{0}^{*}\left(m_{1}, \sigma^{2}, \lambda_{3}, \lambda_{4}\right)+\lambda_{1}^{*}\left(m_{1}, \sigma^{2}, \lambda_{3}, \lambda_{4}\right) y_{i}\right. \\
& \left.+\lambda_{2}^{*}\left(m_{1}, \sigma^{2}, \lambda_{3}, \lambda_{4}\right) y_{i}^{2}+\lambda_{3} y_{i}^{3}+\lambda_{4} y_{i}^{4}\right]
\end{aligned}
$$

and, therefore, the objective function of Definition 2 is equivalent to:

$$
\sum_{i=1}^{n} \log f\left(y_{i} \mid m_{1}\left(x_{i}, \theta_{1}\right), \sigma^{2}\left(x_{i}, \theta_{2}\right), \lambda_{3}\left(x_{i}, \tilde{\theta}_{n}\right), \lambda_{4}\left(x_{i}, \tilde{\theta}_{n}\right)\right)
$$

since the terms $\lambda_{3}\left(x_{i}, \tilde{\theta}_{n}\right) y_{i}^{3}+\lambda_{4}\left(x_{i}, \tilde{\theta}_{n}\right) y_{i}^{4}$ do not depend on $\left(\theta_{1}, \theta_{2}\right)$. The method is called QGPML2 because only $y_{i}$ and $y_{i}^{2}$ are involved, and it is clearly an example of a Quasi-Generalized M-estimator.

Moreover, we obtain the following important property:

Proposition 8. The QGPML2 estimator $\left(\hat{\theta}_{1 n}, \hat{\theta}_{2 n}\right)$ is asymptotically equivalent to the unfeasible estimator based on the maximization of

$$
\begin{aligned}
L_{n 0}^{(2)}\left(\theta_{1}, \theta_{2}\right) & =\sum_{i=1}^{n}\left\{\lambda_{0}^{*}\left[m_{1}\left(x_{i}, \theta_{1}\right), \sigma^{2}\left(x_{i}, \theta_{2}\right), \lambda_{3}\left(x_{i}, \theta_{0}\right), \lambda_{4}\left(x_{i}, \theta_{0}\right)\right]\right. \\
& +\lambda_{1}^{*}\left[m_{1}\left(x_{i}, \theta_{1}\right), \sigma^{2}\left(x_{i}, \theta_{2}\right), \lambda_{3}\left(x_{i}, \theta_{0}\right), \lambda_{4}\left(x_{i}, \theta_{0}\right)\right] y_{i} \\
& \left.+\lambda_{2}^{*}\left[m_{1}\left(x_{i}, \theta_{1}\right), \sigma^{2}\left(x_{i}, \theta_{2}\right), \lambda_{3}\left(x_{i}, \theta_{0}\right), \lambda_{4}\left(x_{i}, \theta_{0}\right)\right] y_{i}^{2}\right\} .
\end{aligned}
$$


Proof. According to the result given in Equation (2.11), we have to check that:

$$
E_{0}\left[\frac{\partial^{2}}{\partial\left(\begin{array}{c}
m_{1} \\
\sigma^{2}
\end{array}\right) \partial\left(\begin{array}{c}
\lambda_{3} \\
\lambda_{4}
\end{array}\right)}\left(\lambda_{0}^{*}+\lambda_{1}^{*} Y_{i}+\lambda_{2}^{*} Y_{i}^{2}\right) \mid X\right]=0 .
$$

Differentiating $\log f\left(y_{i} \mid x_{i} ; m_{1}, \sigma^{2}, \lambda_{3}, \lambda_{4}\right)$ with respect to $m_{1}$ and $\sigma^{2}$ and then taking the expectation we get:

$$
\frac{\partial \lambda_{0}^{*}}{\partial\left(\begin{array}{c}
m_{1} \\
\sigma^{2}
\end{array}\right)}+\frac{\partial \lambda_{1}^{*}}{\partial\left(\begin{array}{c}
m_{1} \\
\sigma^{2}
\end{array}\right)} m_{1}+\frac{\partial \lambda_{2}^{*}}{\partial\left(\begin{array}{c}
m_{1} \\
\sigma^{2}
\end{array}\right)} m_{2}=0,
$$

for any $\left(m_{1}, \sigma^{2}, \lambda_{3}, \lambda_{4}\right)$. Therefore, differentiating further with respect to $\lambda_{3}$ and $\lambda_{4}$, we still get zero.

Proposition 9. The QGPML2 estimator $\left(\hat{\theta}_{1 n}, \hat{\theta}_{2 n}\right)$ is consistent, asymptotically normal, and the asymptotic distribution of $\sqrt{n}\left[\left(\hat{\theta}_{1 n}, \hat{\theta}_{2 n}\right)-\left(\theta_{10}, \theta_{20}\right)\right]$ is $N\left(0, B\left(\theta_{0}\right)\right)$ with

$$
\begin{aligned}
& B\left(\theta_{0}\right)=\left\{E _ { X } \left[\left(\begin{array}{cc}
\frac{\partial m_{1}\left(X, \theta_{10}\right)}{\partial \theta_{1}} & 2 \frac{\partial m_{1}\left(X, \theta_{10}\right)}{\partial \theta_{1}} m_{1}\left(X, \theta_{10}\right) \\
0 & \frac{\partial \sigma^{2}\left(X, \theta_{20}\right)}{\partial \theta_{2}}
\end{array}\right) \Omega_{1}^{-1}\left(X, \theta_{0}\right)\right.\right. \\
& \left.\left.\times\left(\begin{array}{cc}
\frac{\partial m_{1}\left(X, \theta_{10}\right)}{\partial \theta_{1}^{\prime}} & 0 \\
2 \frac{\partial m_{1}\left(X, \theta_{10}\right)}{\partial \theta_{1}^{\prime}} m_{1}\left(X, \theta_{10}\right) & \frac{\partial \sigma^{2}\left(X, \theta_{20}\right)}{\partial \theta_{2}^{\prime}}
\end{array}\right)\right]\right\}^{-1} .
\end{aligned}
$$

and

$$
\Omega_{1}\left(X, \theta_{0}\right)=V_{0}\left[\begin{array}{cc}
Y & X \\
Y^{2} &
\end{array}\right] .
$$

Proof. See Appendix B.

It is easily seen that $B\left(\theta_{0}\right)$ is equal to the semi-parametric efficiency bound based on the first two conditional moments.

From consistent estimators of the asymptotic variance-covariance matrix $B\left(\theta_{0}\right)$, we can deduce Wald and score tests, as well as asymptotic confidence regions.

We also note that, from the proof of Proposition 9, we know that the matrices $\tilde{I}$ and $\tilde{J}$ are equal, and therefore we can also use Likelihood-ratio type tests [see Gourieroux and Monfort 1995b, chapter 18]. More precisely, denoting by $\hat{\theta}_{1 n}^{0}$ and $\hat{\theta}_{2 n}^{0}$ the constrained QGMPL2 estimators obtained by maximizing $L_{n}^{(2)}\left(\theta_{1}, \theta_{2}\right)$ under the null, we can use the test statistic:

$$
\xi_{n}^{R}=2\left[L_{n}^{(2)}\left(\hat{\theta}_{1 n}, \hat{\theta}_{2 n}\right)-L_{n}^{(2)}\left(\hat{\theta}_{1 n}^{0}, \hat{\theta}_{2 n}^{0}\right)\right] .
$$

If the null is $g\left(\theta_{1}, \theta_{2}\right)=0$ where $g$ is an $r$-dimensional vector, then, under the null, $\xi_{n}^{R}$ is asymptotically distributed as a $\chi^{2}(r)$. If $\left(\theta_{1}^{\prime}, \theta_{2}^{\prime}\right)^{\prime}$ is a $p$-dimensional 
vector and the null is of the form $\theta_{1}=h_{1}(\gamma)$ and $\theta_{2}=h_{2}(\gamma)$, where $\gamma$ is a $q$-dimensional vector, then under the null, $\xi_{n}^{R}$ is asymptotically distributed as a $\chi^{2}(p-q)$.

\section{Numerical Implementation}

The implementation of the PML4 and QGPML2 methods necessitates the numerical algorithms that will be described in this section. To this end, let us first introduce the useful notion of a canonical quartic family.

\subsection{The Canonical Quartic Family.}

The PML4 method requires the computation of $\lambda=\left(\lambda_{1}, \cdots, \lambda_{4}\right) \in \Lambda^{*}=$ $\mathbb{R}^{3} \times \mathbb{R}^{-*}$ given $\nu=\left(m_{1}, \sigma^{2}, s, k\right) \in D^{*}$, where $D^{*}=D-\left\{\left(m, \sigma^{2}, s, k\right), s=0\right.$, $k \geq 3\}$ has been defined in Section 2.2. Although the mapping between $\Lambda^{*}$ and $D^{*}$ has been shown to be one to one, it is well know (see Maasoumi 1993, and Ormoneit and White 1999) that the numerical computation of $\lambda$ given $\nu$ is delicate. Our contribution to the problem is to show that the computation of this fourvariate function boils down to the computation of a two variates function, thanks to the introduction of the canonical quartic family.

The following results will be useful:

Proposition 10. i) The quartic family $\left\{Q(\lambda), \lambda \in \Lambda^{*}\right\}$ is globally invariant by any linear mapping $L_{a, b}(y)=(y-a) / b, a \in \mathbb{R}, b>0$.

ii) An equivalence relation is obtained in $\left\{Q(\lambda), \lambda \in \Lambda^{*}\right\}$ by imposing the equality of the skewness and kurtosis.

iii) An equivalent class is obtained by considering the image of any given element of the class, by all the linear mappings $L_{a, b}(y), a \in \mathbb{R}, b>0$.

Proof. i) the p.d.f. of the image of $Q(\lambda)$ by $L_{a, b}(y)$ is

$$
\exp \left[\log b+\lambda_{0}+\lambda_{1}(a+b z)+\lambda_{2}(a+b z)^{2}+\lambda_{3}(a+b z)^{3}+\lambda_{4}(a+b z)^{4}\right],
$$

which is equal to the p.d.f. of $Q\left(\lambda^{*}\right)$ with

$$
\begin{aligned}
& \lambda_{0}^{*}=\log b+\lambda_{0}+\lambda_{1} a+\lambda_{2} a^{2}+\lambda_{3} a^{3}+\lambda_{4} a^{4}, \\
& \lambda_{1}^{*}=\left(\lambda_{1}+2 \lambda_{2} a+3 \lambda_{3} a^{2}+4 \lambda_{4} a^{3}\right) b, \\
& \lambda_{2}^{*}=\left(\lambda_{2}+3 \lambda_{3} a+4 \lambda_{4} a^{2}\right) b^{2}, \\
& \lambda_{3}^{*}=\left(\lambda_{3}+4 \lambda_{4} a\right) b^{3}, \\
& \lambda_{4}^{*}=\lambda_{4} b^{4} .
\end{aligned}
$$

ii) is obvious and iii) is proved by first noting that the image of a distribution $Q(\lambda)$ by $L_{a, b}(y)$ has the same skewness and kurtosis as $Q(\lambda)$ and, second, that any element of a given equivalence class is obtained as the image of any other 
element of the same class by the linear mapping $L_{a, b}(y)$ in which $a$ and $b$ have been adjusted to get the appropriate mean and variance.

It is clear from the formulae given in i) that in any equivalence class we can find an element for which $\lambda_{4}^{*}=-1$ and $\lambda_{3}^{*}=0$, by starting from any $Q(\lambda)$ of the class and taking its image by $L_{a, b}(y)$ with $b=\left(-\lambda_{4}\right)^{-1 / 4}, a=-\lambda_{3} /\left(4 \lambda_{4}\right)$. Moreover, such an element is unique since an other one would be obtained from the first as the image by a linear mapping which is obviously the identity since for both distributions, we should have $\lambda_{4}=-1$ and $\lambda_{3}=0$. This leads to the following definition:

Definition 3. The canonical quartic family $Q^{*}(\alpha, \beta)$ is defined by the family of probability density functions:

$$
\exp \left[\alpha_{0}(\alpha, \beta)+\alpha z+\beta z^{2}-z^{4}\right]
$$

where $(\alpha, \beta) \in \mathbb{R}^{2}$.

The previous results immediately give the following corollary:

Corollary 3. The canonical quartic family $\left\{Q^{*}(\alpha, \beta)\right.$, where $\left.(\alpha, \beta) \in \mathbb{R}^{2}\right\}$ can be parametrized by $(s, k) \in D^{*}$.

It is now clear that the computation of $\lambda=\left(\lambda_{1}, \cdots, \lambda_{4}\right)$ for a given $\nu=$ $\left(m_{1}, \sigma^{2}, s, k\right)$ can be done in two steps. First compute the appropriate $(\alpha, \beta)$ corresponding to $(s, k)$, second find the linear mapping such that the image of $Q^{*}(\alpha, \beta)$ by this mapping has a mean and a variance equal to $\left(m_{1}, \sigma^{2}\right)$, and the image of $Q^{*}(\alpha, \beta)$ by this mapping gives the required $Q(\lambda)$.

The first step necessitates to solve a non-linear two dimensional system. Once $Q^{*}(\alpha, \beta)$ is obtained, the computation of its mean $m_{1}^{*}$ and its variance $\sigma^{* 2}$ is straightforward as well as the computation of $(a, b)$ defined by the system $m_{1}=$ $a+b m_{1}^{*}, \sigma=b \sigma^{*}$. Finally, once the pair $(a, b)$ is known, we immediately have $\lambda_{4}=-b^{-4}$ and $\lambda_{3}=-4 \lambda_{4} a=4 a b^{-4}$. The remaining parameters $\lambda_{0}, \lambda_{1}, \lambda_{2}$ are easily obtained from the first three equations of the system given in the proof of Proposition 10, in which $\lambda_{0}^{*}=\alpha_{0}(\alpha, \beta), \lambda_{1}^{*}=\alpha, \lambda_{2}^{*}=\beta$, since this system is linear recursive in $\lambda_{0}, \lambda_{1}$, and $\lambda_{2}$, yielding

$$
\begin{aligned}
& \lambda_{2}=\beta / b^{2}-3 a \lambda_{3}-6 \lambda_{4} a^{2}, \\
& \lambda_{1}=\alpha / b-2 \lambda_{2} a-3 \lambda_{3} a^{2}-4 \lambda_{4} a^{3}, \\
& \lambda_{0}=\alpha_{0}(\alpha, \beta)-\log b-\left(\lambda_{1} a+\lambda_{2} a^{2}+\lambda_{3} a^{3}+\lambda_{4} a^{4}\right) .
\end{aligned}
$$




\subsection{Computation of the Functions $\alpha(s, k), \beta(s, k)$ Using the Gauss-Freud}

\section{Method.}

In subsection 6.1 we have seen that a key step in the construction of an exponential quartic density is the computation of $\alpha$ and $\beta$ for a given pair of skewness and kurtosis, $(s, k)$.

Using the notation:

$$
q(z ; \alpha, \beta)=\exp \left(\alpha z+\beta z^{2}-z^{4}\right),
$$

we have to compute the integrals:

$$
I_{j}(\alpha, \beta)=\int_{-\infty}^{\infty} z^{j} q(z ; \alpha, \beta) d z, \quad j=0, \cdots, 4 .
$$

Once these integrals are known, we easily get the moments:

$$
m_{j}(\alpha, \beta)=I_{j}(\alpha, \beta) / I_{0}(\alpha, \beta), \quad j=1, \cdots, 4,
$$

and, therefore, $s(\alpha, \beta), k(\alpha, \beta)$. Finally, we have to minimize in $(\alpha, \beta)$ the distance:

$$
[s-s(\alpha, \beta)]^{2}+[k-k(\alpha, \beta)]^{2} .
$$

It is well known that the computation of the parameters for such a problem may be difficult. The basic reason for this is that the function $q(z ; \alpha, \beta)$ may have two maxima for very different values of $z$, and it may take very small values in a large area between these two values of $z$. Furthermore, one of the maxima may be far out in the tails and yet contribute a relatively important probability mass. This implies that integration methods of the Newton type, based on an equidistant grid, are inadequate. More precisely, approximations of the form:

$$
\hat{I}_{j}(\alpha, \beta)=\delta \sum_{i=0}^{N} z_{i}^{j} \exp \left(\alpha z_{i}+\beta z_{i}^{2}-z_{i}^{4}\right),
$$

with $z_{i}-z_{i-1}=\delta$ and $i=1, \cdots, N$, or even improvements thereof, such as Simpson's scheme, may necessitate very large values for $N,-z_{0}$, and $z_{N}$ to achieve acceptable precision. Typically, we would have to take values like $N=20^{\prime} 000$, and $-z_{0}=z_{N}=80$, which makes the optimization of (6.6) very difficult. In addition, "smarter" integration techniques, based on the Gauss-Lagrange scheme, may be problematic since such a scheme requires first a transform of $\mathbb{R}$ into $(-1,1)$, by the logistic map. This transform essentially varies in a neighborhood of the origin, and it tends to lose information contained in the tails. As a consequence, such schemes, even when performed with a large number of abscissas, tend to be inaccurate even for relatively low values of kurtosis. For this reason, we adopt the Gauss-Freud method [see Freud (1986) for the seminal work and Levin and Lubinsky (2000) 
for a recent research-monograph], which is designed to accurately approximate integrals of the kind:

$$
\int_{-\infty}^{\infty} f(z) \exp \left(-z^{4}\right) d z
$$

This method leads to approximations of the form:

$$
I_{j}^{*}(\alpha, \beta)=\sum_{i=0}^{N} z_{i}^{j} \exp \left(\alpha z_{i}+\beta z_{i}^{2}\right) w_{i},
$$

where the abscissa $z_{i}$ and the weights $w_{i}$ are very precisely adapted to the shape of the function to be integrated. Further details on how the $z_{i}$ and the $w_{i}$ may be computed may be found in Gautschi (2004, Part 1).2 Thus, the proposed algorithm has two advantages over the other numerical methods: it uses results on numerical integration specifically related to the integration problem and, moreover, the calculation of parameters involves computing only two parameters, resulting in a significant gain in time. We performed all of the numerical integrations using $N=100 .^{3}$

\subsection{Implementation of the PML4 Method.}

In this section we synthesize the previous sections by presenting an algorithm that describes the computation of $\lambda_{0}, \lambda_{1}, \lambda_{2}, \lambda_{3}, \lambda_{4}$ corresponding to a given $m=$ $\left(m_{1}, m_{2}, m_{3}, m_{4}\right)^{\prime}$.

(1) Compute $s(m), k(m) .{ }^{4}$

(2) Find the pair of $(\alpha, \beta)$ corresponding to $s(m), k(m)$, using the method of Section 6.2.

\footnotetext{
${ }^{2}$ Essentially, the weights $w_{j}$ and abscissa $x_{j}$ can be obtained as eigenvectors and eigenvalues of a Jacobi matrix (see Golub and Welch 1969). This matrix, in turn, requires a sequence of parameters for which a stable estimation algorithm has been proposed by Noschese and Pasquini (1999) for the $\exp \left(-z^{4}\right)$ weight function. Prof. Milovanović implemented this algorithm and made the resulting parameters available to the public via the website of Prof. Gautschi. On this website, one may find the file: coefffreud4.txt under www.cs . purdue.edu/archives $/ 2001 /$ wxg/tables. To obtain the $x_{j}$ and $w_{j}$, we use his routine Gauss.m to be found under: www.cs.purdue.edu/archives/2002/wxg/codes .

${ }^{3}$ The time required to compute for given skewness and kurtosis the parameters $\alpha$ and $\beta$ represents currently a limitation for the application of these methods to large conditional models. One possibility to circumvent this computation consists of computing the $\alpha$ and $\beta$ once and for all and then use some interpolation scheme. We leave such an optimization of the program to further research.

${ }^{4}$ Skewness and kurtosis are given, respectively, by

$$
s(m)=\frac{m_{3}-3 m_{2} m_{1}+2 m_{1}^{3}}{\left(m_{2}-m_{1}^{2}\right)^{3 / 2}}, \quad k(m)=\frac{m_{4}-4 m_{3} m_{1}+6 m_{2} m_{1}^{2}-3 m_{1}^{4}}{\left(m_{2}-m_{1}^{2}\right)^{2}} .
$$
}


(3) Compute the mean $m_{1}^{*}(\alpha, \beta)$ and the variance $\sigma^{2 *}(\alpha, \beta)$ corresponding to the canonical pdf $Q^{*}(\alpha, \beta)$.

(4) The linear transform $Y=a+b Z, b>0$, gives $m_{1}=a+b m_{1}^{*}(\alpha, \beta)$, and $\sigma=b \sigma^{*}(\alpha, \beta)$, where $\sigma^{2}=m_{2}-m_{1}^{2}$. That is, $b=\sigma / \sigma^{*}(\alpha, \beta)$ and $a=m_{1}-b m_{1}^{*}(\alpha, \beta)$.

(5) Compute $\lambda_{4}=-(b)^{-4}, \lambda_{3}=-4 \lambda_{4} a$, and use equations (6.2), (6.3), and (6.4) to get $\lambda_{0}, \lambda_{1}, \lambda_{2}$.

We wish to emphasize that, whereas the previous literature (be it in econometrics, physics, or chemistry) involved the resolution of a non-linear system with four unknowns, our algorithm only involves the resolution of non-linear system with two unknowns. The rest involves elementary algebra. For this reason, our approach will not only be numerically more stable but also significantly faster.

\subsection{Implementation of the QGPML2 Method.}

The numerical problem is the following: given any admissible value of $\left(m_{1}, \sigma^{2}\right.$, $\left.\lambda_{3}, \lambda_{4}\right)$, compute $\lambda_{i}^{*}\left(m_{1}, \sigma^{2}, \lambda_{3}, \lambda_{4}\right)$, for $i=0,1,2$.

According to this approach, it is necessary to allow for densities of any given mean and variance. However, the numerical integration scheme uses the kernel $\exp \left(-z^{4}\right)$, a symmetric kernel that weights those observations in a neighborhood of 0 . We expect that this may create numerical difficulties for random variables whose mean is distant from 0 . For this reason, we consider a computation strategy of the $\lambda_{i}^{*}$ where, in a preliminary step, observations are studentized. ${ }^{5}$

Thus, instead of considering the pdf,

$$
\exp \left[\lambda_{0}^{*}+\lambda_{1}^{*} y+\lambda_{2}^{*} y^{2}+\lambda_{3} y^{3}+\lambda_{4} y^{4}\right]
$$

it is useful to characterize the associated density, which has a mean of zero and a variance of 1 . This density is related to the previous one by the linear transformation $Y=m_{1}+\sigma Z$, where $Z$ represents a random variable with mean 0 and variance 1 . The corresponding density, which will be called the Studentized exponential quartic, is written as:

$$
\exp \left[\delta_{0}+\delta_{1} z+\delta_{2} z^{2}+\delta_{3} z^{3}+\delta_{4} z^{4}\right]
$$

We have the relations $\delta_{4}=\sigma^{4} \lambda_{4}<0$ and $\delta_{3}=\sigma^{3} \lambda_{3}+\frac{4 m_{1}}{\sigma} \delta_{4}$. Since, in the QGPML2 approach, $m_{1}, \sigma^{2}, \lambda_{3}, \lambda_{4}$ are given, the parameters $\delta_{3}, \delta_{4}$ are also given. Once $\delta_{0}, \delta_{1}, \delta_{2}$ corresponding to a zero mean and unit variance have been obtained

\footnotetext{
${ }^{5}$ This studentization is not required for PML4 where the mean $m_{1}^{*}$ (and variances $\left.\left(\sigma^{*}\right)^{2}\right)$ turn out to be close to 0 (1).
} 
using the method described below, one may revert to the initial parameters using:

$$
\begin{aligned}
& \lambda_{0}^{*}=\left[\sigma^{4} \delta_{0}-m_{1} \sigma^{3} \delta_{1}+m_{1}^{2} \sigma^{2} \delta_{2}-m_{1}^{3} \sigma \delta_{3}+m_{1}^{4} \delta_{4}\right] / \sigma^{4}-\log (\sigma), \\
& \lambda_{1}^{*}=\left[\sigma^{3} \delta_{1}-2 m_{1} \sigma^{2} \delta_{2}+3 m_{1}^{2} \sigma \delta_{3}-4 m_{1}^{3} \delta_{4}\right] / \sigma^{4}, \\
& \lambda_{2}^{*}=\left[\sigma^{2} \delta_{2}-3 m_{1} \sigma \delta_{3}+6 m_{1}^{2} \delta_{4}\right] / \sigma^{4} .
\end{aligned}
$$

Using the notation,

$$
q\left(z ; \delta_{1}, \delta_{2}\right)=\exp \left[\delta_{1} z+\delta_{2} z^{2}+\delta_{3} z^{3}+\delta_{4} z^{4}\right]
$$

we have to compute the integrals:

$$
I_{j}\left(\delta_{1}, \delta_{2}\right)=\int_{-\infty}^{\infty} z^{j} q\left(z ; \delta_{1}, \delta_{2}\right) d z, \quad j=0, \cdots, 2
$$

Using the change of variable $u=\left(-\delta_{4}\right)^{1 / 4} z$, these integrals become:

$$
\begin{gathered}
I_{j}\left(\delta_{1}, \delta_{2}\right)=\int_{u=-\infty}^{+\infty}\left(\left(-\delta_{4}\right)^{-1 / 4} u\right)^{j} \exp \left[\delta_{0}+\delta_{1}\left(-\delta_{4}\right)^{-1 / 4} u+\delta_{2}\left(\left(-\delta_{4}\right)^{-1 / 4} u\right)^{2}+\right. \\
\left.\delta_{3}\left(\left(-\delta_{4}\right)^{-1 / 4} u\right)^{3}-u^{4}\right]\left(-\delta_{4}\right)^{-1 / 4} d u \quad j=0, \cdots, 2 .
\end{gathered}
$$

Now, the kernel $\exp \left(-u^{4}\right)$ appears again, and we may use the Gauss-Freud method outlined in Section 6.2. Once these integrals are efficiently evaluated, we may compute the moments:

$$
m_{j}\left(\delta_{1}, \delta_{2}\right)=I_{j}\left(\delta_{1}, \delta_{2}\right) / I_{0}\left(\delta_{1}, \delta_{2}\right), \quad j=1,2 \text {. }
$$

The parameters $\delta_{1}$ and $\delta_{2}$ are obtained by minimizing the distance:

$$
\left[m_{1}\left(\delta_{1}, \delta_{2}\right)\right]^{2}+\left[\sigma^{2}\left(\delta_{1}, \delta_{2}\right)-1\right]^{2}
$$

where $\sigma^{2}\left(\delta_{1}, \delta_{2}\right)=m_{2}\left(\delta_{1}, \delta_{2}\right)-m_{1}^{2}\left(\delta_{1}, \delta_{2}\right)$. Eventually, $\delta_{0}=-\log I_{0}\left(\delta_{1}, \delta_{2}\right)$.

\section{NumERICAL EXAMPLES}

In this section, after discussing the computation of the parameters of the quartic exponential for given moments of order 1 to 4, we will discuss several Monte-Carlo exercises demonstrating the usefulness of the methods at hand. The examples we want to discuss are 1) a comparison between various estimation techniques for small samples, 2) a study of the performance of PML4 in the case of misspecification, and 3) an illustration of QGPML2. 


\subsection{Computation of the Quartic Exponential.}

As discussed in Section 6, feasibility of the PML4 estimation hinges on the ability to efficiently compute the parameters $\lambda_{0}, \cdots, \lambda_{4}$ of the quartic exponential for given $m_{1}, m_{2}, m_{3}, m_{4}$. Ormoneit and White (1999) attribute to Agmon et al. (1981) the first attempts to compute the "correct" $\lambda^{*}$. Agmon et al. (1981) considered the maximization of entropy under moment constraints, that is the socalled primal problem. They computed the integrations using the Gauss-Lagrange scheme after mapping the domain of integration $(-\infty, \infty)$ into $(-1,1)$. Zellner and Highfield (1988) propose computation of the $\lambda^{*}$ by seeking the zeros of the first order conditions that result from the entropy maximization (i.e. the dual approach). Maasoumi (1983) reported difficulties with this method that Ormoneit and White (1999) corroborate. Ormoneit and White (1999) also map the domain $(-\infty, \infty)$ into $(-1,1)$, and they use the Gauss-Lagrange scheme. Moreover, they feed intelligent starting values into their optimization and stabilize the computation of the exponential to avoid numerical overflow. Last but not least, to our knowledge, they are the first ones in the literature to have acknowledged numerical difficulties in the $\lambda$ parameter computation along the segment $s=0, k>3$. Indeed, we know that no density exists for this segment, based on theoretical grounds as discussed earlier.

Our method hinges instead on obtaining the parameters $\alpha$ and $\beta$ of the canonical form for given skewness and kurtosis. In this section, we wish to discuss the precision of these computations. ${ }^{6}$ In order to evaluate the algorithm which gives the parameters $\alpha$ and $\beta$ of the canonical form, we considered a grid covering the range of values of kurtosis from 1.5 to 20. For each value of kurtosis, $k$, we considered a grid for skewness ranging from 0.1 to $\sqrt{k-1}-0.1$. For each point of this grid, say $s, k$, we computed $\alpha$ and $\beta$ as described in Section 6.2 and recomputed the associated skewness and kurtosis, say $\tilde{s}, \tilde{k} .^{7}$ In Figure 1, circles represent the points of the skewness-kurtosis grid for which we evaluated the parameters and, + symbols represent those points for which the distance $D=(s-\tilde{s})^{2}+(k-\tilde{k})^{2}>10^{-5}$.

This figure demonstrates several interesting phenomena 1) Even though, based on theoretical grounds, no density can exist on the segment $(s=0, k \geq 3)$, it is

\footnotetext{
${ }^{6}$ All of the programming was performed in the MATLAB environment. We implemented the code on both Mac OS X and Windows Vista machines. All of the simulations were performed on a PC with an Intel quadricore processor running four MATLAB clones in parallel. To increase the speed of the computations, we transcribed the central part of the programs into the $\mathrm{C}$ language and called it via a MEX interface.

${ }^{7}$ We perform the required minimization using the Nelder-Meade approach.
} 
still possible to obtain a density for parameters close to the excluded segment. 2) Even for very large values of kurtosis (limited in the figure to 20), we obtain a large range of values of skewness for which a highly accurate density may be obtained. We also constructed a similar graph where kurtosis was allowed to took values up to 150 . We find that, even for a kurtosis of 150, the range of skewness, where $D<10^{-5}$, ranges from 2.5 to 12.1 , still a very respectable domain.

Many of the difficulties encountered in earlier attempts at such calculations disappear in our approach. The linear transform $Y=a+b Z$ allows us to write the exponential quartic in terms of $\exp \left(-z^{4}\right)$. Then, by replacing $\exp \left(-z^{4}\right)$ by well-behaved discrete weights (this leads to formula (6.7)), we integrate directly over the range $(-\infty, \infty)$, thus obviating the use of the logistic map. Next, we only optimize over two parameters, rather than four. We also feed optimized starting values for $\alpha$ and $\beta$ into the optimizer. These starting values are the $\alpha$ and $\beta$ corresponding to those points in the domain represented in Figure 1 that are closest to some given values of skewness and kurtosis. The skewness and kurtosis, and their associated $\alpha$ and $\beta$,are stored once and for all in some file that is read into memory as the program is initialized.

To further understand some of the difficulties encountered in earlier studies, we obtained the parameters $\lambda_{j}$, for $j=0, \cdots, 4$ for extremely skewed cases, and evaluated the resulting densities at points far out in the tail (say $z=50$ for a centered and reduced density) and still found a small, yet significant probability mass. The logistic map, which transforms $(-\infty, \infty)$ into $(-1,1)$ used in the earlier work, may therefore have 'fudged' the behavior of the density for relatively large values of skewness and kurtosis. We also note that earlier work required many integration points (each evaluation costs time) whereas using abscissas and weights that are made specifically for the $\exp \left(-z^{4}\right)$ weighting function reduces the number of points for which the integrand needs to be evaluated. ${ }^{8}$

As the numerical exercises that follow will demonstrate, the time necessary to compute the required $\alpha$ and $\beta$ parameters is of an order that is suitable for applying the exponential quartic in many econometric problems. The computation of one set of $\alpha$ and $\beta$ requires about 0.015 seconds, allowing for about 66 density constructions per second. It is clear that the method proposed here is not confined to econometrics and may prove useful in other fields as well.

\footnotetext{
${ }^{8}$ We presently do not incorporate a selection rule on the number of abscissas, $N$, which may further decrease the speed of the computation of the $\alpha$ and $\beta$.
} 
Similarly to the protocol described above, in the context of the parameter estimations related to QGPML2, we verified the precision of the computation of $\delta_{i}$, for $i=0, \cdots, 2$ for given $\delta_{3}, \delta_{4}$, yielding a density with mean 0 and variance 1 .

\subsection{A First Experiment.}

The objective of this first experiment is to demonstrate that the PML4 estimation may provide estimates which are superior to either the PML2 or the GMM estimators in an unconditional setting. We first discuss the choice of a data generating process, and then we focus on the estimation techniques. A priori, many distributions could be used for this experiment (Student-t, distributions in the Pearson family, Gamma, etc.) Preliminary work made it clear that a distribution should be chosen from which draws could be obtained in a very rapid manner. For this reason we settled on the family of skewed Laplace distributions, denoted sLD. These distributions have been used to price options in the context of extreme return realizations, for example, by Gourieroux and Monfort (2006). This family of distributions has three parameters, $b_{0}>0, b_{1}>0$, and $c$, and its pdf is defined by:

$$
f\left(z ; b_{0}, b_{1}, c\right)=\left\{\begin{array}{cc}
\frac{b_{0} b_{1}}{b_{0}+b_{1}} \exp \left[b_{0}(z-c)\right], & \text { if } z \leq c \\
\frac{b_{0} b_{1}}{b_{0}+b_{1}} \exp \left[-b_{1}(z-c)\right], & \text { if } z>c .
\end{array}\right.
$$

The mean, variance, skewness and kurtosis of this density are given by:

$$
\begin{aligned}
m_{1}\left(c, b_{0}, b_{1}\right) & =E[Y]=c+\frac{1}{b_{1}}-\frac{1}{b_{0}}, \\
\sigma^{2}\left(c, b_{0}, b_{1}\right) & =\operatorname{Var}[Y]=\frac{1}{b_{0}^{2}}+\frac{1}{b_{1}^{2}}, \\
s\left(c, b_{0}, b_{1}\right) & =\frac{2}{\sigma^{3}}\left[\frac{1}{b_{1}^{3}}-\frac{1}{b_{0}^{3}}\right], \\
k\left(c, b_{0}, b_{1}\right) & =\frac{9}{\sigma^{4}}\left[\frac{1}{b_{1}^{4}}+\frac{1}{b_{0}^{4}}\right]+\frac{6}{\sigma^{4} b_{0}^{2} b_{1}^{2}} .
\end{aligned}
$$

Since this density may be viewed as describing a mixture of exponentials, we use the inverse c.d.f. technique to simulate random draws from it.

In this first experiment, we focus on the situation where $c=0$. Indeed, without an additional assumption on one of the parameters of the sLD, we would not be able, in the following, to obtain parameter estimates based on the PML2 principle.

We simulated 10,000 samples, each of a length of either $T=25,50,100$, or 1000 i.i.d. observations. The estimation techniques used were ML, PML2, PML4 and GMM. Let us describe the way in which we implemented these estimations. 
For ML, we maximized for each sample, the log-likelihood obtained from (7.1):

$$
L^{M L}=\sum_{i=1}^{T} \log f\left(z_{i} ; b_{0}, b_{1}\right)
$$

For PML2, we considered the objective function:

$$
L^{P M L 2}=-T \log \sigma\left(b_{0}, b_{1}\right)-\frac{1}{2} \sum_{i=1}^{T}\left(\frac{z_{i}-m_{1}\left(b_{0}, b_{1}\right)}{\sigma\left(b_{0}, b_{1}\right)}\right)^{2} .
$$

For PML4, we formed the objective function:

$$
L^{P M L 4}=\sum_{i=1}^{T} \lambda_{0}+\lambda_{1} y_{i}+\lambda_{2} y_{i}^{2}+\lambda_{3} y_{i}^{3}+\lambda_{4} y_{i}^{4},
$$

where the parameters $\lambda_{0}, \cdots, \lambda_{4}$ were computed as described in section 6.3 for $\left(m_{1}\left(b_{0}, b_{1}\right), \sigma\left(b_{0}, b_{1}\right)^{2}, s\left(b_{0}, b_{1}\right), k\left(b_{0}, b_{1}\right)\right)$.

For GMM, we defined, (see Hansen (1982)), the $4 \times 1$ vector,

$$
X_{i}\left(b_{0}, b_{1}\right)=\left[\begin{array}{c}
z_{i}-m_{1}\left(b_{0}, b_{1}\right) \\
z_{i}^{2}-m_{1}^{2}\left(b_{0}, b_{1}\right)-\sigma^{2}\left(b_{0}, b_{1}\right) \\
\left(\frac{z_{i}-m_{1}\left(b_{0}, b_{1}\right)}{\sigma\left(b_{0}, b_{1}\right)}\right)^{3}-s\left(b_{0}, b_{1}\right) \\
\left(\frac{z_{i}-m_{1}\left(b_{0}, b_{1}\right)}{\sigma\left(b_{0}, b_{1}\right)}\right)^{4}-k\left(b_{0}, b_{1}\right)
\end{array}\right]
$$

and considered the distance:

$$
J=g_{T}\left(b_{0}, b_{1}\right)^{\prime} S^{-1} g_{T}\left(b_{0}, b_{1}\right), \text { where } g_{T}\left(b_{0}, b_{1}\right)=\frac{1}{T} \sum_{i=1}^{T} X_{i}\left(b_{0}, b_{1}\right)
$$

The GMM estimates were obtained as those parameters minimizing the distance $J$. The matrix $S$ that appears in the distance was obtained by using, as a first step, the identity matrix, and as a second step, the asymptotic variance-covariance matrix. Thus, the GMM estimates are asymptotically optimal in the sense that they reach the semiparametric bound. However, it is well know that the preliminary estimation of the optimal matrix $S$ may induce some biases in finite samples.

We performed the simulation using $\left(b_{0}, b_{1}\right)=(2.41,1.30) .{ }^{9}$ This point corresponds to the moments $\left(m_{1}, m_{2}, s, k\right)=(1.30,0.35,1.15,6.91)$. All estimations were performed using the MATLAB fminsearch optimizer and John d'Errico's fminsearchbnd's extension. The bounds that we impose for the ML and GMM

\footnotetext{
${ }^{9}$ We also used other points, but the results are very similar to the ones reported here.
} 
estimations are wide and only serve to stabilize the optimization, they do not affect the final results. ${ }^{10}$

Table 1 displays several statistics for 10,000 simulations and for various sample sizes. The main result that this Table conveys is that, for all sample sizes considered, the MSE of the ML estimation dominates, as expected, over all other methods. However, we find that the PML4 technique yields estimates with an MSE up to less than half of the one for PML2. As far as the GMM are concerned, the total MSE is always the largest, especially when the number of observations is small, confirming the bad behavior of GMM in this setting. This suggests that for situations where the econometrician has no prior information on the skewed distribution to use for the estimation, the PML4 technique may be a most useful one.

\subsection{A Second Experiment.}

Many dynamic models are specified in the following way:

$$
y_{t}=m\left(y_{\underline{t-1}}, \theta\right)+\sigma\left(\underline{y_{t-1}}, \theta\right) \varepsilon_{t},
$$

where $m\left(y_{\underline{t-1}}, \theta\right)$ and $\sigma\left(y_{\underline{t-1}}, \theta\right)$ are functions of the past values $y_{t-1}=\left\{y_{t-1}, \cdots\right.$ ,$\left.y_{1}\right\}$ of $y_{t}$, and of a parameter $\theta,\left\{\varepsilon_{t}\right\}$ being a zero mean, unit variance white noise process. In this kind of setting, the conditional skewness and kurtosis of $y_{t}$ given $y_{\underline{t-1}}$ are the same as those of $\varepsilon_{t}$, and, therefore, do not depend on $y_{\underline{t-1}}$. This is a strong information that the PML4 method is able to take into account while not assuming any specific distribution of $\varepsilon_{t}$. For this reason, we can expect that the PML4 methods will perform better than, for instance, PML2 methods, and misspecified ML methods. We consider as setting

$$
\begin{aligned}
y_{t} & =\sigma_{t} \varepsilon_{t}, \\
\sigma_{t}^{2} & =\omega+\alpha\left(y_{t-1}\right)^{2}+\beta \sigma_{t-1}^{2}, \\
\varepsilon_{t} & \sim \operatorname{MixN}\left(\mu_{1}, \mu_{2}, \sigma_{1}, \sigma_{2}, p\right),
\end{aligned}
$$

where $\operatorname{MixN}\left(\mu_{1}, \mu_{2}, \sigma_{1}, \sigma_{2}, p\right)$ corresponds to the mixture of normals distribution where a first normal distribution with parameters $\mu_{1}$ as mean, and $\sigma_{1}^{2}$ as variance, gets drawn with a probability of $p$ and where a second normal distribution with corresponding parameters $\mu_{2}$ and $\sigma_{2}^{2}$ gets drawn with a probability of $1-p$. The

\footnotetext{
${ }^{10}$ The constraints are $0.001 \leq b_{0} \leq 10$ and $0.00001 \leq b_{1} \leq 5$. In general, in nonlinear estimations, it may occur that the optimizer proposes values of skewness and over-kurtosis that are such that the $Q(\lambda)$ density may not be computed. In such cases, one may issue a warning and force the function to be optimized to return a penalty value telling the optimizer to seek parameters in other regions.
} 
family of two normals can reach any set of mean, variance, skewness, kurtosis, and this, moreover, in a non-unique way. We choose the parameters of MixN such that its expected value, $\mu$, is nil and its variance, $\sigma^{2}$, equal to one. We select several values of skewness and kurtosis for MixN.

Furthermore, to investigate the robustness with respect to the choice of $\varepsilon_{t}$ we consider two data generating processes. In the first case, we chose the MixN in such a way that it maximizes the entropy under the moment constraints. Formally, we select that density for MixN, say $p$, which maximizes $-\int_{-\infty}^{+\infty} p(x) \log p(x) d x$. In the second case, we impose the same standard deviation for both distributions of the mixture, that is $\sigma_{1}=\sigma_{2} \cdot{ }^{11}$

The parameters of the variance dynamic $\sigma_{t}^{2}$ are set to typical values $(\omega=0.1$, $\alpha=0.05$, and $\beta=0.9$ ). For each simulation, we compute misspecified ML estimates as well as the PML2 and PML4 estimates of $\omega, \alpha$, and $\beta$. As misspecified ML we consider the estimations where $\varepsilon_{t}$ is distributed as a (symmetric) Student-t or as an (asymmetric) skewed Student-t. ${ }^{12}$ The skewed Student-t has two parameters: first $\lambda$ characterizing the asymmetry of the density and $\eta$ characterizing the tail-fatness. ${ }^{13}$

The Student-t is misspecified since it is a symmetric distribution whereas the simulated innovations are skewed. The skewed Student-t density is also misspecified since its density does not belong to the exponential family. From the work of Newey and Steigerwald (1997) it is know that if one performs likelihood estimations involving asymmetric distributions, it is desirable to include an additional parameter, say $\zeta$, for the location of the innovation density. Formally, we estimate

\footnotetext{
${ }^{11}$ More details on the construction of these processes are available upon request. The formulae on how to obtain these mixtures may be found in Titterington et al. (1985).

${ }^{12}$ The Student-t only has one parameter, $\nu$, describing the fatness of the tails.

${ }^{13}$ Hansen's Student-t distribution is defined by

$$
g(z \mid \eta, \lambda)=\left\{\begin{array}{cc}
b c\left(1+\frac{1}{\eta-2}\left(\frac{b z+a}{1-\lambda}\right)^{2}\right)^{-\frac{\eta+1}{2}} & \text { if } z<-a / b \\
b c\left(1+\frac{1}{\eta-2}\left(\frac{b z+a}{1+\lambda}\right)^{2}\right)^{-\frac{\eta+1}{2}} & \text { if } z \geq-a / b
\end{array}\right.
$$

where

$$
a \equiv 4 \lambda c \frac{\eta-2}{\eta-1}, \quad b^{2} \equiv 1+3 \lambda^{2}-a^{2}, \quad c \equiv \frac{\Gamma\left(\frac{\eta+1}{2}\right)}{\sqrt{\pi(\eta-2)} \Gamma\left(\frac{\eta}{2}\right)} .
$$

The use of a Student-t is a very popular assumption to model in a parcimonious manner large outliers, see Bollerslev and Wooldridge (1992). The choice of a skewed Student-t has been developed by Hansen (1994) and independently by Fernandez and Steel (1998). It has been successfully used by Jondeau and Rockinger (2003), in the context of finance.
} 
a model where

$$
\frac{y_{t}}{\sigma_{t}}-\zeta \sim \varepsilon_{t}, \text { and } \sigma_{t}^{2}=\omega+\alpha\left(y_{t-1}\right)^{2}+\beta \sigma_{t-1}^{2} .
$$

In the simulations, we use $M=10,000$ replications of samples containing $T=2000$ observations. Such sample sizes are typical for finance applications.

A general remark is that the estimation involving the PML2 or the two estimations involving the Student-t take between 0.07 and 0.7 seconds. The PML4 estimation takes between 1.7 seconds (for kurtic and moderately skewed data) up to 5.7 seconds (for very lightly or very heavily skewed distributions). ${ }^{14}$

Table 2 displays the results from the various simulations. The upper part of that table contains the parameters used for MixN, whereas the lower part contains the Root-Mean-Squared errors, defined as

$$
R M S=\sqrt{\frac{1}{M} \sum_{i=1}^{M}\left(\hat{\omega}^{i}-\omega\right)^{2}}+\sqrt{\frac{1}{M} \sum_{i=1}^{M}\left(\hat{\alpha}^{i}-\alpha\right)^{2}}+\sqrt{\frac{1}{M} \sum_{i=1}^{M}\left(\hat{\beta}^{i}-\beta\right)^{2}},
$$

where the symbol $i$ denotes the number of a simulation.

Columns numbered 1 and 4 consider the cases where the distributions are kurtic, yet nearly symmetric. Columns 2 and 5 correspond to cases where the DGP density is moderately skewed. The skewed Student-t can reach the given skewness. Columns 3 and 6 correspond to cases where the density is heavily skewed. The skewed Student-t can not reach the given theoretical values. Columns 7 and 8 correspond to cases where the mixture of normals assumes a same standard deviation.

Inspection of the last line (corresponding to the RMS) of the GARCH-PML4 model shows that for all the cases under consideration, the PML4 model outperforms all other estimation techniques. We also notice that for very skewed distributions, the GARCH-Skt model does well, whereas those GARCH models based on a symmetric distribution generate much larger RMS.

Last, we wish to check the robustness of the simulation results if the innovation distribution is changed. To do so we replace the entropy-maximizing mixture of normals with one where standard deviations are the same for each distribution. The comparison of the results obtained for the last two columns with columns 5

\footnotetext{
${ }^{14}$ All estimations were performed using identical lower and upper bounds on the parameters. We used as starting values for PML2 $\left(\omega=10^{-3}, \alpha=0.15\right.$, and $\left.\beta=0.8\right)$. We used the PML2 parameter estimates as starting values for all subsequent estimations. We also used the estimated values of skewness and overkurtosis of PML2 as starting values for PML4. In all cases, the degree of freedom of the Student-t is imposed to be larger than 4 , which is equivalent to imposing existence of kurtosis for the Student-t.
} 
and 6 reveals nearly identical figures (at least for the first two decimals). Again, PML4 results as the distributional assumption yielding the lowest RMS.

We also checked the RMS at the level of the individual parameters and found that if an overall RMS was better for one model than for the other, the RMS improvements came from all parameters.

As this experiment suggests, in the case of skewed and kurtic data, great care needs to be exercised if one uses ML estimation with a risk of misspecification. The PML4 method appears to be much more robust.

7.4. A Third Experiment. In the previous examples, we demonstrated the usefulness of the PML4 technique, first in an IID case, second in a dynamic case. In both cases the skewness and kurtosis (conditional in the second example) were constant. Here, we consider a case where they depend on an exogenous variable. There are many situations where the modeling of the variation in higher moments may be of importance per se. For instance, Hansen (1994) considered a GARCH model with time varying skewness and kurtosis.

A generic model that captures variation in the higher moments is given by:

$$
y_{i}=\mu+\sigma \varepsilon_{i},
$$

where $\varepsilon_{i} \sim D\left(0,1, s\left(x_{i}\right), k^{*}\left(x_{i}\right)\right)$,

$$
\begin{aligned}
s\left(x_{i}\right) & =0.5+a x_{i}, \\
k^{*}\left(x_{i}\right) & =2+b x_{i}, b>0, \\
x_{i} & \sim U[1 / 2,3 / 2], \text { i.i.d. }
\end{aligned}
$$

In the second line, $D$ stands for some distribution where skewness and overkurtosis depends on some exogenous variable, $x_{i}$. The next two lines specify how skewness and over-kurtosis are parametrized. We recall that the kurtosis, $k$, is related to the over-kurtosis, $k^{*}$, by $k=1+s^{2}+k^{*}$. For the simulations, we take the lower moments to be $\mu=0$ and $\sigma=1$. Furthermore, we take $a=1$ and $b=2$. As long as the condition $b>0$ is imposed in the numerical computation, the model will be well defined. The intercepts 0.5 and 2 in the specification of $s\left(x_{i}\right)$ and $k^{*}\left(x_{i}\right)$ guarantee that the distribution will be skewed (here $s>1$ ) and fat-tailed (here $k>4$ ).

The $D$ distribution that we choose is the mixture of two normal distributions with identical variances $\operatorname{MixN}\left(\mu_{1}, \mu_{2}, \sigma, \sigma, p\right)$ already discussed in the previous section. In the Monte-Carlo exercise, we simulate 1,500 samples, each with $T=$ 
100 observations. The estimations require between about 60 and 340 sec., with an average time of about 130 sec. $^{15}$

Table 3 contains the statistics associated with the various estimations. As this Table demonstrates, even though the numerical complexity behind the PML4 computation is significant, this method may be actually implemented even in a Monte Carlo framework with many replications (here 1,500). With the feasibility of the method already demonstrated, we may now turn to the interpretation of the statistics. We first note that the parameters tend to be estimated rather well if one uses the average of the estimates. The MSE of the mean $\mu$ is 0.05. The MSE of the parameter $b$ that describes the kurtosis of the distribution takes a higher value. We find that the parameter estimates are skewed and kurtic, and we note that the MSE of the parameter estimates increases with the order of the moment that a given parameter describes.

We conclude this section by noticing that our method may obviously be used in real applications, for models which may have more parameters, since it then has to be estimated only once.

\subsection{A Fourth Experiment: QGPML2.}

To validate the QGPML2 approach, we consider as DGP the observation $\left(y_{i}, x_{i}\right)$ generated by:

$$
\begin{aligned}
y_{i} & =a x_{i}+\exp \left(b x_{i}\right) \varepsilon_{i}, \\
u_{i} & \sim U(0,1), \quad \text { i.i.d. } \\
x_{i} & =\left(1+29 u_{i}\right) / 10, \\
\theta_{i} & =\left(1+29 u_{i}\right) \frac{\pi}{180}, \\
\varepsilon_{i} & \sim \operatorname{sLD}\left(\frac{1}{\sin \theta_{i}}, \frac{1}{\cos \theta_{i}}, \sin \theta_{i}-\cos \theta_{i}\right) .
\end{aligned}
$$

The first line specifies the mean and the variance of the model as depending on some exogenous variable $x_{i}$. The second line defines the $u_{i}$ as uniform draws. From this basic source of exogenous randomness, we construct $x_{i}$ as uniform random numbers $U(1 / 10,3)$. The fourth line specifies $\theta_{i}$ as an angle that varies between 1 and 30 degrees. The ratio $\pi / 180$ converts this angle into radians. The last equation specifies that $\varepsilon_{i}$ is distributed according to the skewed Laplace distribution with mean 0 , variance 1 , and known skewness and kurtosis. A similar

\footnotetext{
${ }^{15}$ We also performed several estimations involving samples of size $T=1000$. The time required for each estimation ranged between about 2100 seconds and 2800 seconds.
} 
parametrization was chosen in Subsection 7.3. We select as parameters $a=1$ and $b=1$.

A preliminary simulation revealed that, for this parametrization, the skewness (kurtosis) of the $\varepsilon_{i}$ ranges between 0 and 2 (respectively, 6 and 9).

The QGPML2 estimation is based on the following steps:

(1) Estimate $a$ and $b$ via PML2. This is tantamount to obtaining $a, b$ by maximizing the function:

$$
\sum_{i=1}^{T}-b x_{i}-\frac{1}{2}\left(\frac{y_{i}-a x_{i}}{\exp \left(b x_{i}\right)}\right)^{2} .
$$

(2) Compute the first step estimators $\tilde{\lambda}_{j, i}$ for $j=1, \cdots, 4$ by using the information contained in $s\left(x_{i}\right)$ and $k\left(x_{i}\right)$. This computation is described in section 5.2. Notice that only $\tilde{\lambda}_{3 i}$ and $\tilde{\lambda}_{4 i}$ are used in the next step.

(3) Maximize, with respect to $a$ and $b$, the objective function:

$$
\sum_{i=1}^{T} \lambda_{0, i}^{*}+\lambda_{1, i}^{*} y_{i}+\lambda_{2, i}^{*} y_{i}^{2},
$$

where, $\lambda_{j, i}^{*}=\lambda\left(a x_{i}, \exp \left(b x_{i}\right), \tilde{\lambda}_{3 i}, \tilde{\lambda}_{4 i}\right)$. The computation of the $\lambda_{i, j}^{*}$ may be found in section 6.4. The resulting $a$ and $b$ estimates are the QGPML2 estimates.

Table 4 reports some statistics for the simulations. Each time we use $N=$ 30,000 , a rather large number of simulations to ascertain that the findings are not spurious. We consider samples of size $T=25$ and $T=100 .^{16}$

Inspecting the table reveals that, as expected, the dispersion of the estimates obtained in the larger sample tends to be better. Comparing the dispersion of the parameters between the PML2 estimates and the QGPML2 estimates reveals an improvement when using QGPML2. ${ }^{17}$ For instance, for $T=25$, the improvement of the RMSE of the parameter intervening in the mean, $a$, is $2.6 \%$.

\footnotetext{
${ }^{16}$ Here, we focus on a large number of simulations each involving a relatively small sample. The time required for this simulation could alternatively be devoted to the estimation of a model with either a larger sample or a more complex model structure involving several parameters. We performed some exploratory analysis with samples of size $T=1000$ which requested between 500 and 1300 seconds.

${ }^{17}$ We did not pursue a search for settings where the efficiency gain may be more important, as we simply wished to demonstrate the feasibility of the method here. We leave this pursuit for future research.
} 


\section{Conclusion}

In this paper, we generalize the PML2 and QGPML1 methods proposed in Gourieroux, Monfort, and Trognon (1984). The main objective of these methods was to propose consistent and asymptotically normal estimators of the parameters which appear in the specification of the first two conditional moments, based on the optimization of possibly misspecified likelihood functions.

Here, we extend this approach by considering the first four conditional moments. A key tool is the quartic exponential family. This family allows us to introduce PML4 and QGPML2 estimators, respectively, generalizing PML2 and QGPML1. A complete asymptotic theory is proposed.

Another key issue is the numerical computation of the exponential quartic density parameters for given values of the first four moments. The solution adopted in this paper, which is based on an approach proposed by G. Freud (1986), appears to be very quick and stable, and it solves technical problems, which had been stressed in different strands of the literature, e.g. Maasoumi (1993), Ormoneit and White (1999).

In numerical studies, we not only demonstrate the feasibility of the proposed estimation methods, but also show that PML4 may provide more efficient estimates, in particular for small samples where GMM based estimates may have encountered difficulties. We also consider an example where an econometrician uses either a misspecified ML model or the PML4 model. In that case, the PML4 model demonstrates superior results. Lastly, we show the feasibility of the QGPML2 estimation, and in that context, we again prove gains in efficiency.

Our estimation method may prove useful in many econometric applications that involve non-Gaussianity of some random variable. For instance, Holly (2009) has recently studied risk adjustment schemes for health care expenditures. A key feature of this problem is the large skewness and kurtosis of the conditional distributions of these expenditures given demographic characteristics and the prior health status of policy holders. The PML4 method is obviously well suited to tackle this kind of problem since it incorporates the additional information on third and fourth order conditional moments and does not necessitate parametric assumptions on the conditional distribution. Moreover, the results in Holly (2009) show that the estimations only based on PML2 methods introduce a substantial amount of bias in the risk adjustment 
Beyond this, the proposed numerical techniques may be of relevance in Bayesian analysis, independent component analysis, and possibly physics, i.e. in situations where non-Gaussian distributions occur. 
Appendix A. Computation of $J\left(\theta_{0}\right)$ And $I\left(\theta_{0}\right)$

$$
\begin{aligned}
J\left(\theta_{0}\right) & =-\frac{\partial^{2} \varphi_{\infty}\left(\theta, P_{0}\right)}{\partial \theta \partial \theta^{\prime}} \\
& =-E_{X}\left[\frac{\partial^{2} \lambda_{0}\left(X, \theta_{0}\right)}{\partial \theta \partial \theta^{\prime}}+\sum_{j=1}^{4} \frac{\partial^{2} \lambda_{j}\left(X, \theta_{0}\right)}{\partial \theta \partial \theta^{\prime}} m_{j 0}\right],
\end{aligned}
$$

We have (omitting the variables $X$ and $\theta_{0}$ ):

$$
\begin{aligned}
\frac{\partial \lambda_{j}}{\partial \theta} & =\frac{\partial m^{\prime}}{\partial \theta} \frac{\partial \lambda_{j}}{\partial m}, \quad j=0, \ldots, 4, \\
\frac{\partial^{2} \lambda_{j}}{\partial \theta \partial \theta^{\prime}} & =\frac{\partial m^{\prime}}{\partial \theta} \frac{\partial^{2} \lambda_{j}}{\partial m \partial m^{\prime}} \frac{\partial m}{\partial \theta^{\prime}}+\sum_{k=1}^{4} \frac{\partial \lambda_{j}}{\partial m_{k}} \frac{\partial^{2} m_{k}}{\partial \theta \partial \theta^{\prime}} \\
J\left(\theta_{0}\right) & =-E_{X}\left\{\frac{\partial m^{\prime}}{\partial \theta}\left[\frac{\partial^{2} \lambda_{0}}{\partial m \partial m^{\prime}}+\sum_{j=1}^{4} \frac{\partial^{2} \lambda_{j}}{\partial m \partial m^{\prime}} m_{j 0}\right] \frac{\partial m}{\partial \theta^{\prime}}\right\} \\
& -\left\{\sum_{k=1}^{4}\left[\frac{\partial \lambda_{0}}{\partial m_{k}}+\frac{\partial \lambda_{1}}{\partial m_{k}} m_{10}+\cdots+\frac{\partial \lambda_{4}}{\partial m_{k}} m_{40}\right] \frac{\partial^{2} m_{k}}{\partial \theta \partial \theta^{\prime}}\right\},
\end{aligned}
$$

and using Proposition 1, Corollary 1, and Proposition 2:

$$
\begin{aligned}
J\left(\theta_{0}\right) & =E_{X}\left[\frac{\partial m^{\prime}}{\partial \theta} \frac{\partial \lambda^{\prime}}{\partial m} \frac{\partial m}{\partial \theta^{\prime}}\right] \\
& =E_{X}\left[\frac{\partial m^{\prime}}{\partial \theta} \Sigma^{-1} \frac{\partial m}{\partial \theta^{\prime}}\right] .
\end{aligned}
$$

Similarly,

$$
\begin{aligned}
I\left(\theta_{0}\right) & =E_{X} E_{0}\left[\frac{\partial \varphi\left(Y, X, \theta_{0}\right)}{\partial \theta} \frac{\partial \varphi\left(Y, X, \theta_{0}\right)}{\partial \theta^{\prime}}\right] \\
& =E_{X} E_{0}\left[\left(\frac{\partial \lambda_{0}}{\partial \theta}+\frac{\partial \lambda^{\prime}}{\partial \theta} T\right)\left(\frac{\partial \lambda_{0}}{\partial \theta^{\prime}}+T^{\prime} \frac{\partial \lambda}{\partial \theta^{\prime}}\right)\right],
\end{aligned}
$$

where $\varphi(Y, X, \theta)=\sum_{j=0}^{4} \lambda_{j}(X, \theta) Y^{j}$, and $T^{\prime}=\left(Y, Y^{2}, Y^{3}, Y^{4}\right)$.

Using Proposition 1, we have (omitting the variables $X$ and $\theta_{0}$ ):

$$
\begin{aligned}
I\left(\theta_{0}\right) & =E_{X} E_{0}\left[\frac{\partial \lambda^{\prime}}{\partial \theta}(T-m)(T-m)^{\prime} \frac{\partial \lambda}{\partial \theta^{\prime}}\right] \\
& =E_{X}\left(\frac{\partial \lambda^{\prime}}{\partial \theta} \Omega \frac{\partial \lambda}{\partial \theta^{\prime}}\right) \\
& =E_{X}\left(\frac{\partial m^{\prime}}{\partial \theta} \frac{\partial \lambda^{\prime}}{\partial m} \Omega \frac{\partial \lambda}{\partial m^{\prime}} \frac{\partial m}{\partial \theta^{\prime}}\right) \\
& =E_{X}\left(\frac{\partial m^{\prime}}{\partial \theta} \Sigma^{-1} \Omega \Sigma^{-1} \frac{\partial m}{\partial \theta^{\prime}}\right) .
\end{aligned}
$$




\section{Appendix B. Asymptotic Behavior of the QGPML2}

We focus on the proof of the asymptotic normality since the proof of the consistency is similar to that of the PML4.

\section{B.1. Preliminaries.}

Let us consider the quartic family parametrized by $\xi^{\prime}=\left(m_{1}, \sigma^{2}, \lambda_{3}, \lambda_{4}\right)$. If we fix $\lambda_{3}, \lambda_{4}$ to a given value of $\lambda_{3}^{*}, \lambda_{4}^{*}$, then we obtain a family, indexed by $\left(m_{1}, \sigma^{2}\right)$, with log-density:

$$
\sum_{j=0}^{2} \lambda_{j}^{*}\left(\xi_{12}\right) y^{j}+\lambda_{3}^{*} y^{3}+\lambda_{4}^{*} y^{4},
$$

where $\xi_{12}=\left(m_{1}, \sigma^{2}\right)^{\prime}$ and $\lambda_{j}^{*}\left(\xi_{12}\right)$ is a notation which stands for $\lambda_{j}^{*}\left(m_{1}, \sigma^{2}, \lambda_{3}^{*}, \lambda_{4}^{*}\right)$. It is the log-density of a quadratic exponential family (see GMT (1984)).

Differentiating with respect to $\xi_{12}$, we get

$$
\sum_{j=0}^{2} \frac{\partial \lambda_{j}^{*}}{\partial \xi_{12}} m_{j}=0, \quad\left(\text { with } m_{0}=1\right)
$$

and

$$
\sum_{j=0}^{2} \frac{\partial^{2} \lambda_{j}^{*}}{\partial \xi_{12} \partial \xi_{12}^{\prime}} m_{j}+\frac{\partial \lambda^{*^{\prime}}}{\partial \xi_{12}} \frac{\partial m_{12}}{\partial \xi_{12}^{\prime}}=0,
$$

with $\lambda^{*}=\left(\lambda_{1}^{*}, \lambda_{2}^{*}\right)^{\prime}$ and $m_{12}=\left(m_{1}, m_{2}\right)^{\prime}$.

Moreover,

$$
\frac{\partial m_{12}}{\partial \xi_{12}^{\prime}}=\left(\begin{array}{cc}
1 & 0 \\
2 m_{1} & 1
\end{array}\right) .
$$

The variance-covariance matrix $\Sigma_{1}$ of $\left(Y, Y^{2}\right)^{\prime}$ in this family is easily found. For instance, we note that the pdf with respect to the measure $\mu^{*}$ defined by $d \mu^{*}(y)=\exp \left(\lambda_{3}^{*} y^{3}+\lambda_{4}^{*} y^{4}\right) d y$ is $\exp \left[\lambda_{0}^{*}\left(\xi_{12}\right)+\lambda_{1}^{*}\left(\xi_{12}\right) y+\lambda_{2}^{*}\left(\xi_{12}\right) y^{2}\right]$. Using the parametrization $\left(\lambda_{1}^{*}, \lambda_{2}^{*}\right)$ and the general property of the exponential family given in $2.1(2)$ :

$$
\Sigma_{1}=\frac{\partial m_{12}}{\partial \lambda^{*^{\prime}}}
$$

which, written as a function of $\xi_{12}$, leads to:

$$
\Sigma_{1}=\frac{\partial m_{12}}{\partial \xi_{12}^{\prime}} \frac{\partial \xi_{12}}{\partial \lambda^{*^{\prime}}}
$$

We therefore have:

$$
\frac{\partial \xi_{12}}{\partial \lambda^{*^{\prime}}}=\left(\frac{\partial m_{12}}{\partial \xi_{12}^{\prime}}\right)^{-1} \Sigma_{1}
$$


hence:

$$
\frac{\partial \lambda^{*}}{\partial \xi_{12}^{\prime}}=\Sigma_{1}^{-1} \frac{\partial m_{12}}{\partial \xi_{12}^{\prime}}
$$

\section{B.2. Computation of $\tilde{J}\left(\theta_{0}\right)$.}

In the estimation based on the unfeasible equivalent estimator of the QGPML2, we have, noting $\theta_{12}=\left(\theta_{1}, \theta_{2}\right)$ :

$$
\begin{gathered}
\tilde{J}\left(\theta_{0}\right)=-E_{X}\left\{\sum _ { j = 0 } ^ { 2 } \frac { \partial ^ { 2 } \lambda _ { j } ^ { * } } { \partial \theta _ { 1 2 } \partial \theta _ { 1 2 } ^ { \prime } } \left[m_{1}\left(X, \theta_{10}\right), \sigma^{2}\left(X, \theta_{20}\right),\right.\right. \\
\left.\left.\lambda_{3}\left(X, \theta_{0}\right), \lambda_{4}\left(X, \theta_{0}\right)\right] m_{j}\left(X, \theta_{0}\right)\right\} .
\end{gathered}
$$

But we have also (omitting $X$ and the $\theta$ 's):

$$
\begin{aligned}
\frac{\partial \lambda_{j}^{*}}{\partial \theta_{12}} & =\frac{\partial \xi_{12}^{\prime}}{\partial \theta_{12}} \frac{\partial \lambda_{j}^{*}}{\partial \xi_{12}} \\
\frac{\partial^{2} \lambda_{j}^{*}}{\partial \theta_{12} \partial \theta_{12}^{\prime}} & =\frac{\partial \xi_{12}^{\prime}}{\partial \theta_{12}} \frac{\partial^{2} \lambda_{j}^{*}}{\partial \xi_{12} \partial \xi_{12}^{\prime}} \frac{\partial \xi_{12}}{\partial \theta_{12}^{\prime}}+\sum_{k=1}^{2} \frac{\partial \lambda_{j}^{*}}{\partial \xi_{k}} \frac{\partial^{2} \xi_{k}}{\partial \theta_{12} \partial \theta_{12}^{\prime}} .
\end{aligned}
$$

Therefore,

$$
\begin{aligned}
\tilde{J}\left(\theta_{0}\right) & =-E_{X}\left[\frac{\partial \xi_{12}^{\prime}}{\partial \theta_{12}} \sum_{j=0}^{2} \frac{\partial^{2} \lambda_{j}^{*}}{\partial \xi_{12} \partial \xi_{12}^{\prime}} m_{j} \frac{\partial \xi_{12}}{\partial \theta_{12}^{\prime}}\right] \\
& -E_{X} \sum_{k=1}^{2}\left[\sum_{j=0}^{2} \frac{\partial \lambda_{j}^{*}}{\partial \xi_{k}} m_{j}\right] \frac{\partial^{2} \xi_{k}}{\partial \theta_{12} \partial \theta_{12}^{\prime}},
\end{aligned}
$$

or, using (B.1) and (B.2),

$$
\tilde{J}\left(\theta_{0}\right)=E_{X}\left[\frac{\partial \xi_{12}^{\prime}}{\partial \theta_{12}} \frac{\partial \lambda^{*^{\prime}}}{\partial \xi_{12}} \frac{\partial m_{12}}{\partial \xi_{12}^{\prime}} \frac{\partial \xi_{12}}{\partial \theta_{12}^{\prime}}\right]
$$

or, using (B.4),

$$
\tilde{J}\left(\theta_{0}\right)=E_{X}\left[\frac{\partial \xi_{12}^{\prime}}{\partial \theta_{12}} \frac{\partial m_{12}^{\prime}}{\partial \xi_{12}} \Sigma_{1}^{-1} \frac{\partial m_{12}}{\partial \xi_{12}^{\prime}} \frac{\partial \xi_{12}}{\partial \theta_{12}^{\prime}}\right] .
$$

\section{B.3. Computation of $\tilde{I}\left(\theta_{0}\right)$.}

Letting $T_{1}=\left(Y, Y^{2}\right)^{\prime}$, we have,

$$
\tilde{I}\left(\theta_{0}\right)=E_{X} E_{0}\left[\left(\frac{\partial \lambda_{0}^{*}}{\partial \theta_{12}}+\frac{\partial \lambda^{* \prime}}{\partial \theta_{12}} T_{1}\right)\left(\frac{\partial \lambda_{0}^{*}}{\partial \theta_{12}^{\prime}}+T_{1}^{\prime} \frac{\partial \lambda^{*}}{\partial \theta_{12}^{\prime}}\right)\right],
$$


or, using (B.1),

$$
\begin{aligned}
\tilde{I}\left(\theta_{0}\right) & =E_{X} E_{0}\left[\frac{\partial \lambda^{* \prime}}{\partial \theta_{12}}\left(T_{1}-m_{12}\right)\left(T_{1}-m_{12}\right)^{\prime} \frac{\partial \lambda^{*}}{\partial \theta_{12}^{\prime}}\right] \\
& =E_{X}\left[\frac{\partial \lambda^{* \prime}}{\partial \theta_{12}} \Omega_{1} \frac{\partial \lambda^{*}}{\partial \theta_{12}^{\prime}}\right] .
\end{aligned}
$$

We also have

$$
\frac{\partial \lambda^{*^{\prime}}}{\partial \theta_{12}}=\frac{\partial \xi_{12}^{\prime}}{\partial \theta_{12}} \frac{\partial \lambda^{* \prime}}{\partial \xi_{12}}=\frac{\partial \xi_{12}^{\prime}}{\partial \theta_{12}} \frac{\partial m_{12}^{\prime}}{\partial \xi_{12}} \Sigma_{1}^{-1},
$$

which allows us to write

$$
\tilde{I}\left(\theta_{0}\right)=E_{X}\left[\frac{\partial \xi_{12}^{\prime}}{\partial \theta_{12}} \frac{\partial m_{12}^{\prime}}{\partial \xi_{12}} \Sigma_{1}^{-1} \Omega_{1} \Sigma_{1}^{-1} \frac{\partial m_{12}}{\partial \xi_{12}^{\prime}} \frac{\partial \xi_{12}}{\partial \theta_{12}^{\prime}}\right] .
$$

However, since the moments of order one to four are well specified in the pseudo family, the true conditional variance $\Omega_{1}\left(X, \theta_{0}\right)$ of $T_{1}$ is that corresponding to the model. Namely, they correspond to $\Sigma_{1}\left(X, \theta_{0}\right)$, and we get:

$$
\tilde{I}\left(\theta_{0}\right)=E_{X}\left[\frac{\partial \xi_{12}^{\prime}}{\partial \theta_{12}} \frac{\partial m_{12}^{\prime}}{\partial \xi_{12}} \Omega_{1}^{-1} \frac{\partial m_{12}}{\partial \xi_{12}^{\prime}} \frac{\partial \xi_{12}}{\partial \theta_{12}^{\prime}}\right]
$$

and hence, from (B.5):

$$
\tilde{I}\left(\theta_{0}\right)=\tilde{J}\left(\theta_{0}\right) .
$$

Moreover, using (B.3),

$$
\frac{\partial m_{12}}{\partial \xi_{12}^{\prime}} \frac{\partial \xi_{12}}{\partial \theta_{12}^{\prime}}=\left(\begin{array}{cc}
1 & 0 \\
2 m_{1} & 1
\end{array}\right)\left(\begin{array}{cc}
\frac{\partial m_{1}\left(X, \theta_{1}\right)}{\partial \theta_{1}} & 0 \\
0 & \frac{\partial \sigma^{2}\left(X, \theta_{2}\right)}{\partial \theta_{2}}
\end{array}\right),
$$

and $\tilde{I}\left(\theta_{0}\right)^{-1}=\tilde{J}\left(\theta_{0}\right)^{-1}=B\left(\theta_{0}\right)$, as given in (5.2).

For the sake of simplicity, we have assumed that $m_{1}\left(X, \theta_{1}\right)$ is only a function of $\theta_{1}$ and that $\sigma^{2}\left(X, \theta_{2}\right)$ is only a function of $\theta_{2}$. The result, however, is easily generalized to the case $m_{1}\left(X, \theta_{12}\right), \sigma^{2}\left(X, \theta_{12}\right)$, with the only difference being that $\partial \xi_{12} / \partial \theta_{12}^{\prime}$ is no longer diagonal. 


\section{REFERENCES}

[1] Agmon, N., Y. Alhassid, and R.D. Levine (1979): "An Algorithm for Finding the Distribution of Maximal Entropy," Journal of Computational Physics, 30, 250-259.

[2] Andersen, T.G., and B. Sorenson (1996): "GMM Estimation of a Stochastic Volatility Model: A Monte Carlo Study," Journal of Business 83 Economic Statistics, 14, 328-352.

[3] Altonji, J.G. and Segal, L.M. (1996): "Small-Sample Bias in GMM Estimation of Covariance Structures," Journal of Business \&3 Economic Statistics, 14(3), 353-366.

[4] Arellano-Valle, R.B., and M.G., Genton (2005): "On Fundamental Skew Distributions," Journal of Multivariate Analysis, 96, 93-116.

[5] Barndorff-Nielsen, O.E. (1978): Information and Exponential Families in Statistical Theory. Wiley, Chichester.

[6] Barndorff-Nielsen, O.E. (1997): "Normal Inverse Gaussian Distributions and Stochastic Volatility Modeling," Scandinavian Journal of Statistics, 24, 1-13.

[7] Brown, L.D. (1986): "Fundamentals of Statistical Exponential Families," Institute of Mathematical Statistics, Hayward, California.

[8] Bollerslev, T., and J.M. Wooldridge (1992): "Quasi-Maximum Likelihood Estimation and Inference in Dynamic Models with Time Varying Covariances," Econometric Reviews, 11(2), 143-172.

[9] Chamberlain, G. (1987): "Asymptotic Efficiency in Estimation with Conditional Moment Restrictions," Journal of Econometrics, 34, 305-334.

[10] Doran, H.E., and P. Schmidt (2006): "GMM Estimators with Improved Finite Sample Properties using Principal Components of the Weighting Matrix, with an Application to the Dynamic Panel Data Model," Journal of Econometrics, 133(1), 387-409.

[11] Eberlein, E., and U. Keller (1995): "Hyperbolic Distributions in Finance," Bernoulli, 1, 281-299.

[12] Fernandez, C., and M.F.J. Steel (1998): "On Bayesian Modelling of Fat Tails and Skewness," Journal of the American Statistical Association, 93, 359-371.

[13] Freud, G., (1986): "On the Greatest Zero of an Orthogonal Polynomial, I," Journal of Approximation Theory, 46, 16-24.

[14] Gale D., and H. Nikaido (1965): "The Jacobian Matrix and Global Univalence of Mappings," Mathematische Annalen, 159(2), 81-93.

[15] Gallant A.R. (1987): Nonlinear Statistical Models. John Wiley \& Sons, New York.

[16] Gautschi, W. (2004): Orthogonal Polynomials: Computation and Approximation. Oxford Science Publications, Oxford University Press.

[17] Genton M.G. (2004): "Skew-Elliptical Distributions and Their Applications: A Journey Beyond Normality," Edited vol., Chapman \& Hall/CRC, Boca Raton, Florida.

[18] Golan, A., G. Judge, and D. Miller (1996): Maximum Entropy Econometrics: Robust Estimation with Limited Data. Wiley, Chichester.

[19] Golub, G.H., and J.H. Welch (1969): "Calculation of Gauss Quadrature Rules," Mathematics of Computations, 23(106), 221-230.

[20] Gourieroux C., A. Monfort, and A. Trognon (1984): "Pseudo Maximum Likelihood Methods: Theory," Econometrica, 52(1), 681-700.

[21] Gourieroux C., and A. Monfort (1995a): Statistics and Econometric Models: Volume One. Cambridge University Press, Cambridge.

[22] Gourieroux C., and A. Monfort (1995b): Statistics and Econometric Models: Volume Two. Cambridge University Press, Cambridge.

[23] Gourieroux C., and A. Monfort (2006): "Pricing with Splines," Annales d'Economie et de Statistique, 82, 4-33. 
[24] Hansen, L.P. (1982): "Large Sample Properties of the Generalized Methods of Moments," Econometrica, 50, 1029-1054.

[25] Hansen, B. (1994): "Autoregressive Conditional Density Estimation," International Economic Review, 35, 705-730.

[26] Harvey, C.R., and Siddique, A. (1999): "Autoregressive Conditional Skewness," Journal of Financial and Quantitative Analysis, 34(4), 465-487.

[27] Holly A. (1993): "Asymptotic Theory for Nonlinear Econometric Models: Estimation," in Aart J. de Zeeuw (ed.) Advanced Lectures in Quantitative Economics II, Academic Press.

[28] Holly, A., and Y. Pentsak (2004): "Maximum Likelihood Estimation of the Conditional Mean $E(y \mid x)$ for Skewed Dependent Variables in Four-parameter Families of Distribution," Technical Report, Institute of Health Economics and Management, University of Lausanne.

[29] Holly, A (2009): "Modeling Risk Using Fourth Order Pseudo Maximum Likelihood Methods," mimeo, Institute of Health Economics and Management, University of Lausanne.

[30] Hood, W., and T. Koopmans (1953): "The Estimation of Simultaneous Linear Economic Relationships," in Studies in Econometric Method. New Haven: Yale University Press.

[31] Jondeau, E., and M. Rockinger (2003): "Conditional Volatility, Skewness, and Kurtosis: Existence, Persistence, and Comovements," Journal of Economic Dynamics and Control, 27(10), 1699-1737.

[32] Junk, M. (2000): "Maximum Entropy for Reduced Moment Problems," Mathematical Models and Methods in Applied Sciences, 10(7), 1001-1025.

[33] Kitamura, Y., and M. Stutzer (1997): "An Information-Theoretic Alternative to Generalized Method of Moments Estimation," Econometrica, 65(4), 861-874.

[34] Levin, E., and D.S. Lubinsky (2001): Orthogonal Polynomials for Exponential Weights. CMS Books in Mathematics, Springer-Verlag, New-York.

[35] Manning W., A. Basu, and J. Mullahy (2005): "Generalized Modeling Approaches to Risk Adjustment of Skewed Outcomes Data," Journal of Health Economics, 24, 465-488.

[36] Maasoumi, E. (1993): "A Compendium To Information Theory in Economics and Econometrics," Econometric Reviews, 12(2), 137-181.

[37] Mead, L.R., and N. Papanicolaou (1984): "Maximum Entropy in the Problem of Moments," Journal of Mathematical Physics, 25(8), 2404-2417.

[38] Monfort, A. (1982): Cours de Statistique Mathématique. Economica, Paris.

[39] Newey, W.K. (1990): "Semiparametric Efficiency Bounds," Journal of Applied Econometrics, 5, 99-135.

[40] Noschese S., and L. Pasquini (1999): "On the Nonnegative Solution of a Freud Three-term Recurrence," Journal of Approximation Theory, 99, 54-67.

[41] Ormoneit, D., and H. White (1999): "An Efficient Algorithm to Compute Maximum Entropy Densities," Econometric Reviews, 18(2), 127-140.

[42] Rockafellar, R.T. (1970): Convex Analysis. Princeton University Press.

[43] Sawa, T. (1978): "Information Criteria for Discriminating Among Alternative Regression Models," Econometrica, 46(6), 1273-1291.

[44] Stacy, E. (1962): "A Generalization of Gamma Distribution," Annals of Mathematical Statistics, 33, 1187-1192.

[45] Stacy, E., and G. Mihram (1965): "Parameter Estimation for a Generalized Gamma Distribution," Technometrics, 7, 349-358.

[46] Tauchen, G. (1986): "Statistical Properties of Generalized Method-of-Moments Estimators of Structural Parameters Obtained from Financial Market Data," Journal of Business \& Economic Statistics, 4(4), 397-416.

[47] Titterington, D.M., A.F.M. Smith, and U.E., Makov (1985): Statistical Analysis of Finite Mixture Distributions. John Wiley, Chichester. 
[48] White, H. (1982): "Maximum Likelihood of Misspecified Models," Econometrica, 50(1), $1-25$.

[49] White, H. (1994): Estimation, Inference, and Specification Analysis. Cambridge University Press, Cambridge, UK.

[50] Zellner, A., and R.A. Highfield (1988): "Calculation of Maximum Entropy Distributions and Approximation of Marginal Posterior Distributions," Journal of Econometrics, 37(2), 195-209.

[51] Ziliak, J.P. (1997) : "Efficient Estimation with Panel Data when Instruments are Predetermined: An Empirical Comparison of Moment-Condition Estimators," Journal of Business and Economic Statistics, 15, 419-431. 
$\stackrel{1}{\underline{2}}$

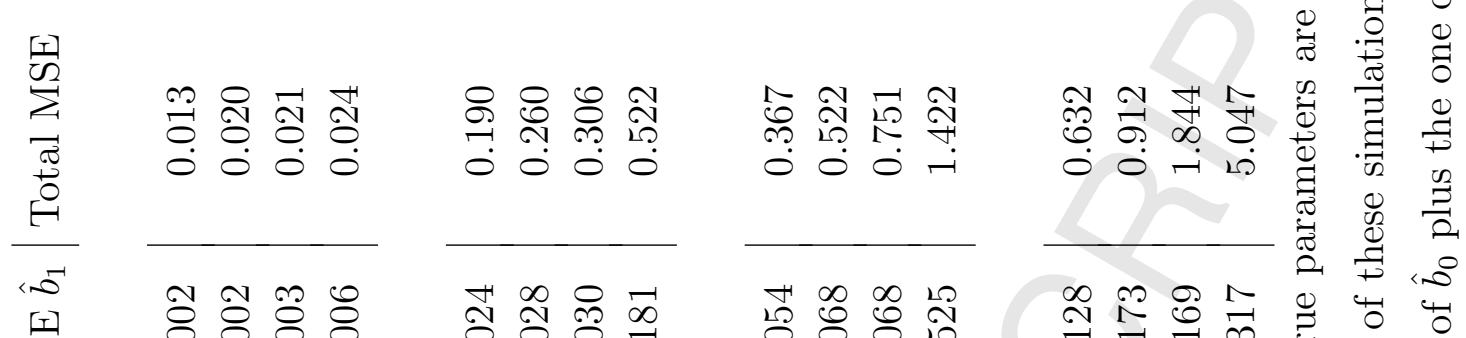

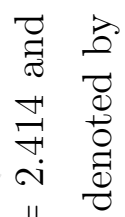

$8 . \frac{\pi}{4}<\frac{5}{4}$

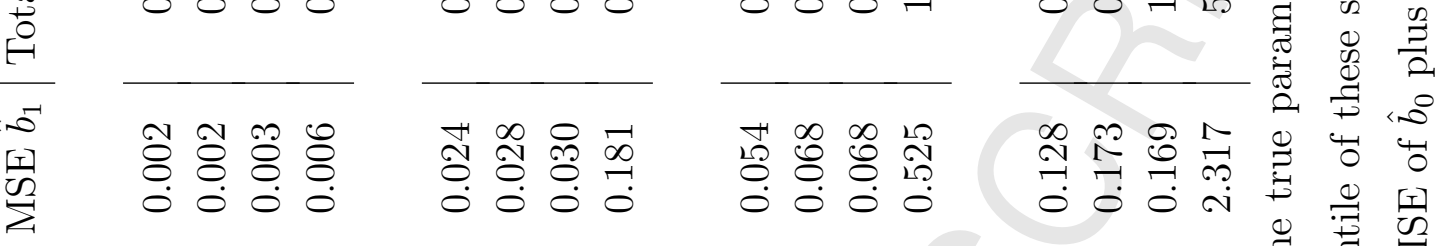

(1)

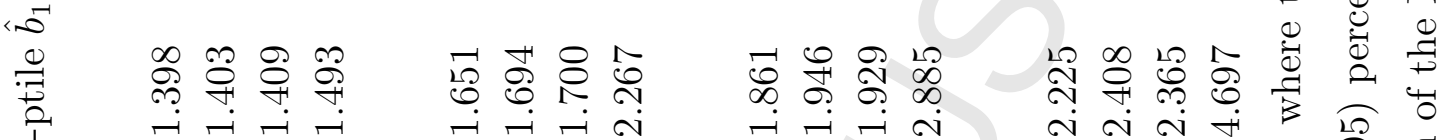

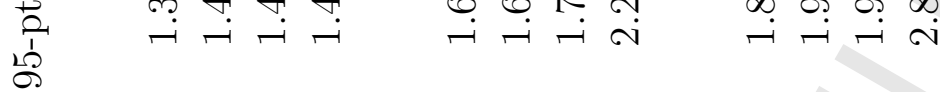

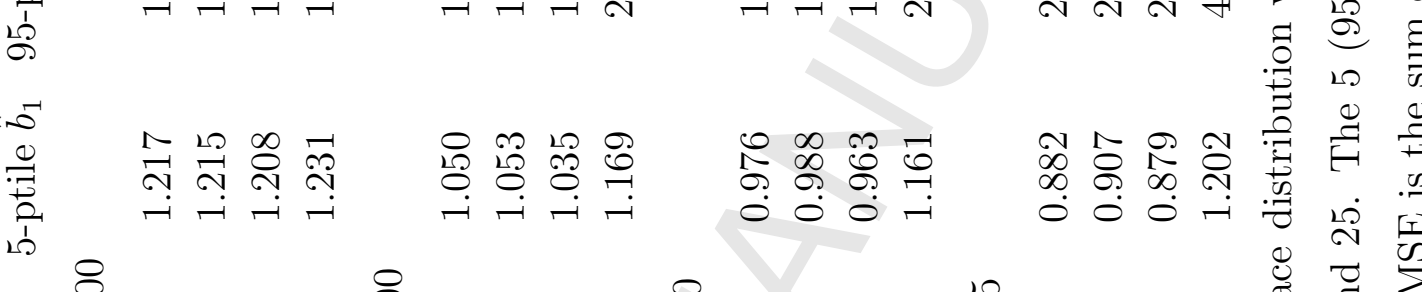

《

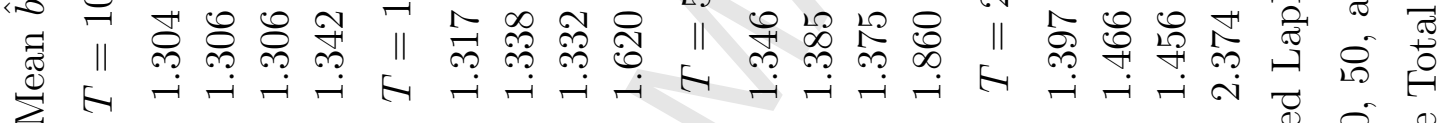

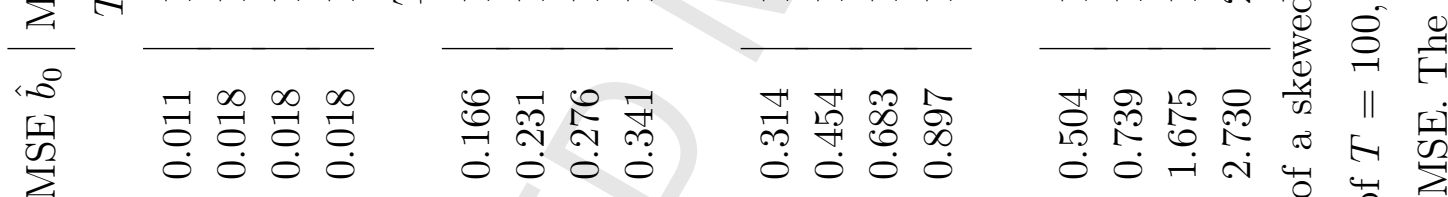

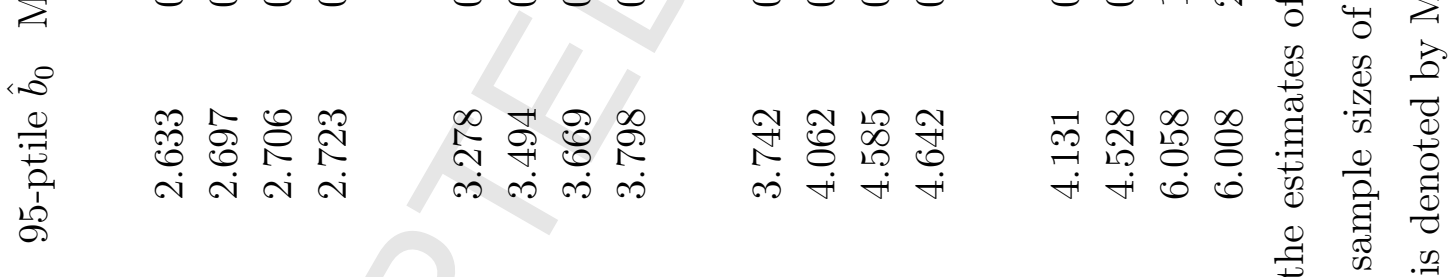

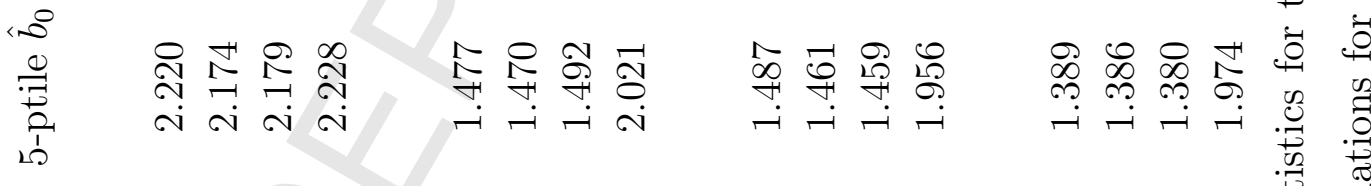

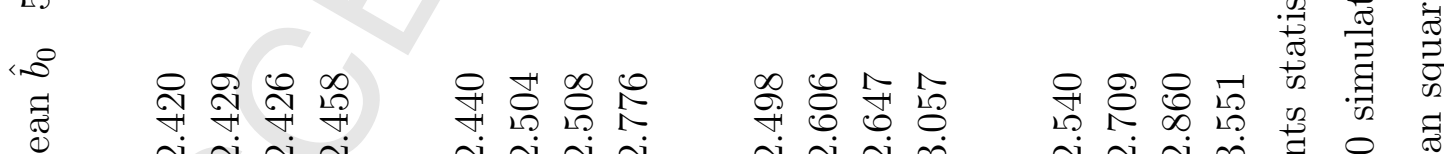

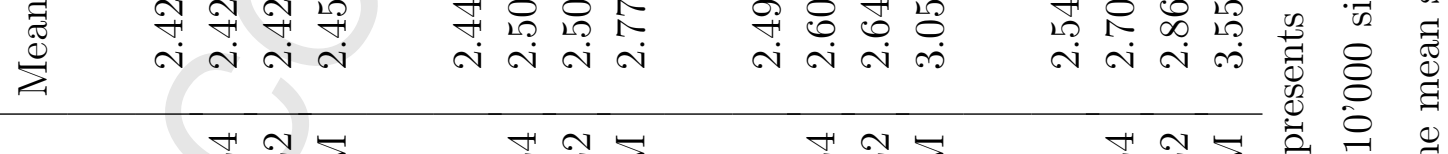

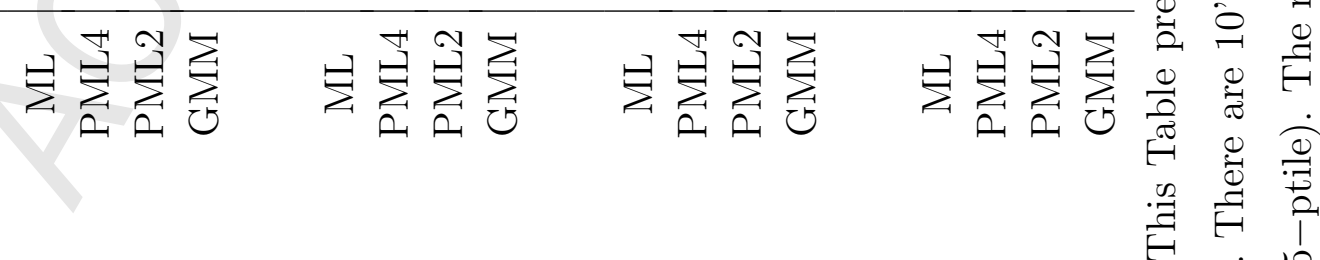

$\ddot{\sim} \dot{\circ} \stackrel{20}{\mathscr{C}}$

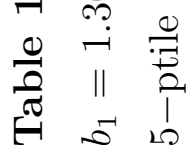




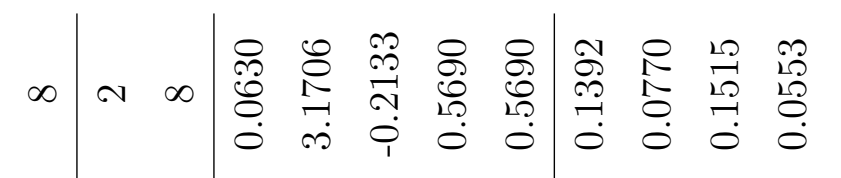

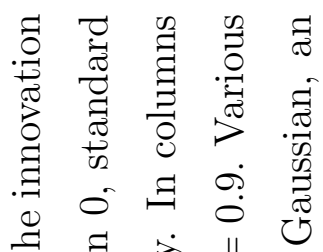

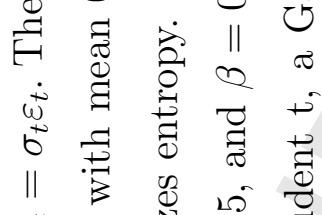

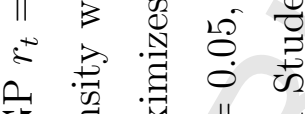

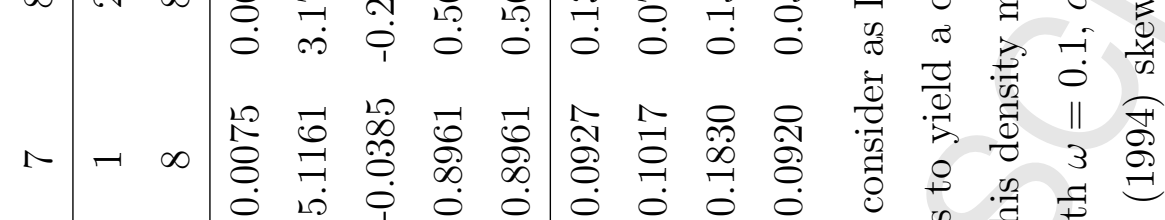

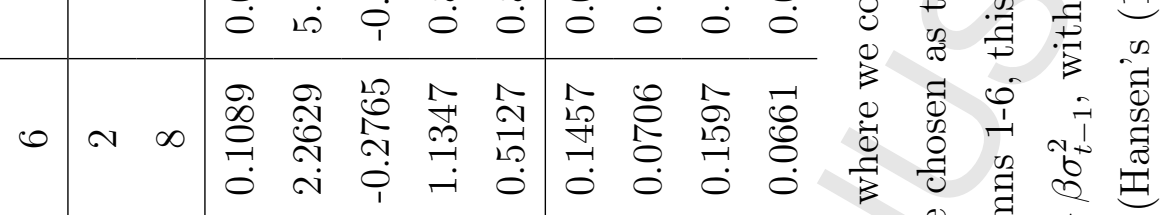

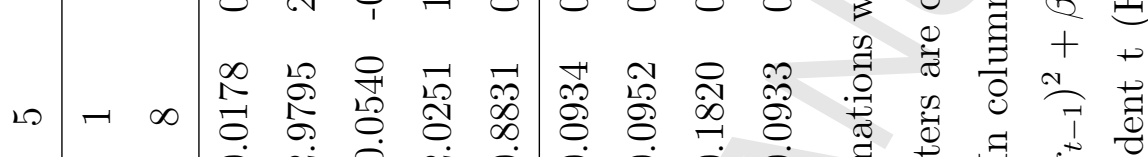

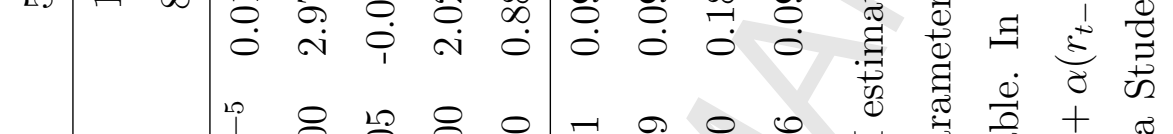

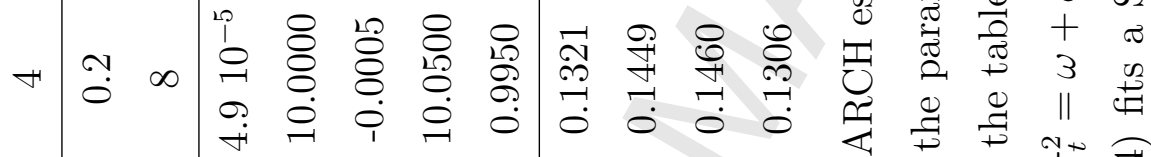

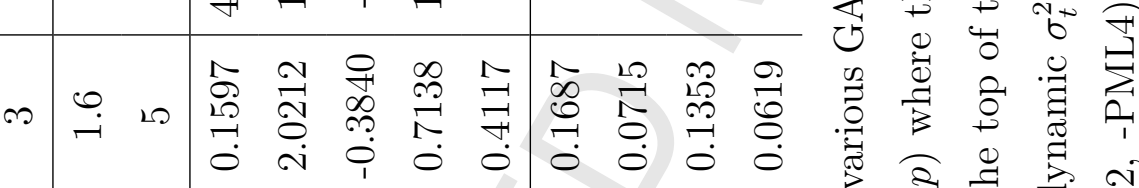

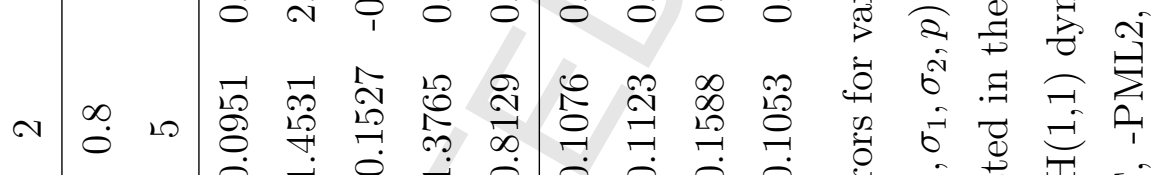

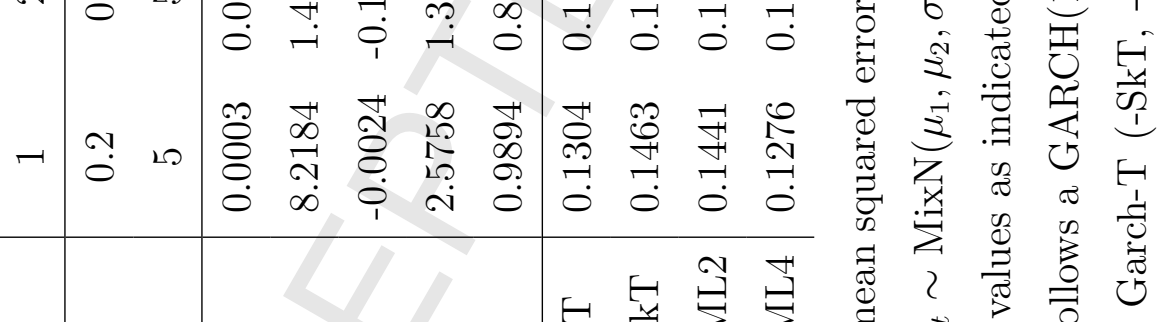

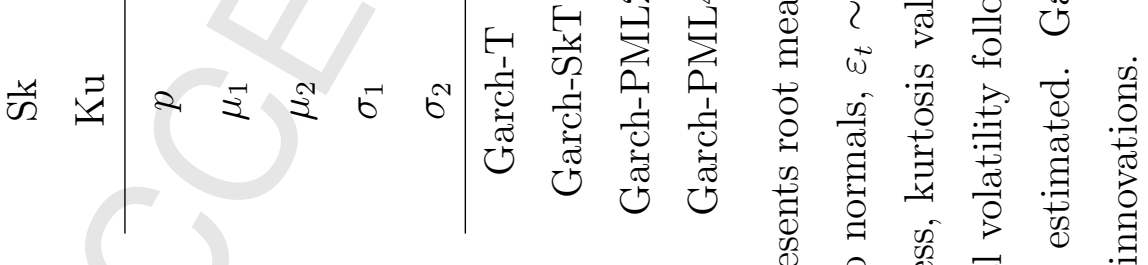

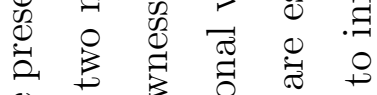

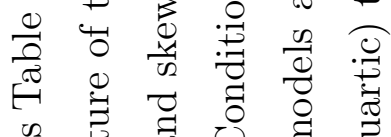

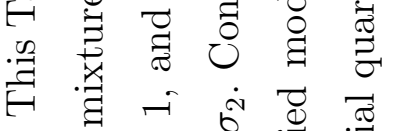

i

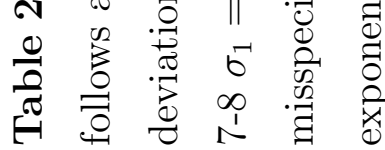




\begin{tabular}{c|cccc} 
& $\mu$ & $\sigma$ & $a$ & $b$ \\
\hline True parameters & 0 & 1 & 1 & 2 \\
\hline Average & 0.006 & 1.015 & 1.109 & 2.510 \\
Std & 0.071 & 0.136 & 0.641 & 2.058 \\
Median & 0.000 & 1.011 & 1.057 & 1.794 \\
Min & -0.277 & 0.695 & 0.000 & 0.000 \\
Max & 0.304 & 1.950 & 4.000 & 7.000 \\
Sk & 0.666 & 0.980 & 1.886 & 1.290 \\
$\sigma_{S k}$ & 0.063 & 0.063 & 0.063 & 0.063 \\
Ku & 5.607 & 6.869 & 9.500 & 3.398 \\
$\sigma_{K u}$ & 0.126 & 0.126 & 0.126 & 0.126 \\
MSE & 0.005 & 0.019 & 0.422 & 4.490
\end{tabular}

Table 3: This Table presents the statistics of 1'500 PML4 estimates of $\mu$, $\sigma, a$ and $b$ as described in the model (7.7). Each sample contained $T=100$ observations. Sk, Ku represent the skewness and kurtosis of the parameters. By $\sigma_{S k}$ and $\sigma_{K u}$ we represent the standard deviation of the skewness and kurtosis estimates. 


\begin{tabular}{c|cc|cc} 
& \multicolumn{4}{|c}{ PML2 } \\
True parameters & \multicolumn{2}{|c}{$T=25$} & $T=100$ \\
\hline Mean & 0.996 & 0.915 & 0.994 & 0.956 \\
STD & 0.567 & 0.249 & 0.401 & 0.177 \\
min & 0.001 & 0.001 & 0.001 & 0.331 \\
max & 4.464 & 2.206 & 2.848 & 1.619 \\
RMSE & 0.567 & 0.263 & 0.401 & 0.182
\end{tabular}

\begin{tabular}{c|cc|cc} 
& \multicolumn{4}{|c}{ QGPML2 } \\
& \multicolumn{2}{|c}{$T=25$} & $T=100$ \\
True parameters & $a=1$ & $b=1$ & $a=1$ & $b=1$ \\
\hline Mean & 0.997 & 0.917 & 0.998 & 0.957 \\
STD & 0.552 & 0.247 & 0.393 & 0.176 \\
$\min$ & 0.001 & 0.001 & 0.001 & 0.330 \\
$\max$ & 3.880 & 2.200 & 2.543 & 1.641 \\
$\Delta$ RMSE (\%) & 2.606 & 0.937 & 2.193 & 0.728
\end{tabular}

Table 4: This Table reports the results of the QGPML2 simulation described in model (7.8). The true parameters are $a=1$, and $b=1$. The RMSE is defined as $\left(\frac{1}{M} \sum_{j=1}^{M}\left(\hat{\theta}^{(j)}-\theta\right)^{2}\right)^{1 / 2}$, where $\theta=a$ or $b$. Here, the superscript $j=1, \cdots, M$ denotes a simulation. We took $M=30^{\prime} 000$. By $\Delta$ RMSE (\%) we denote the percentage gain in the MSE if one uses QGPML2 instead of PML2. 


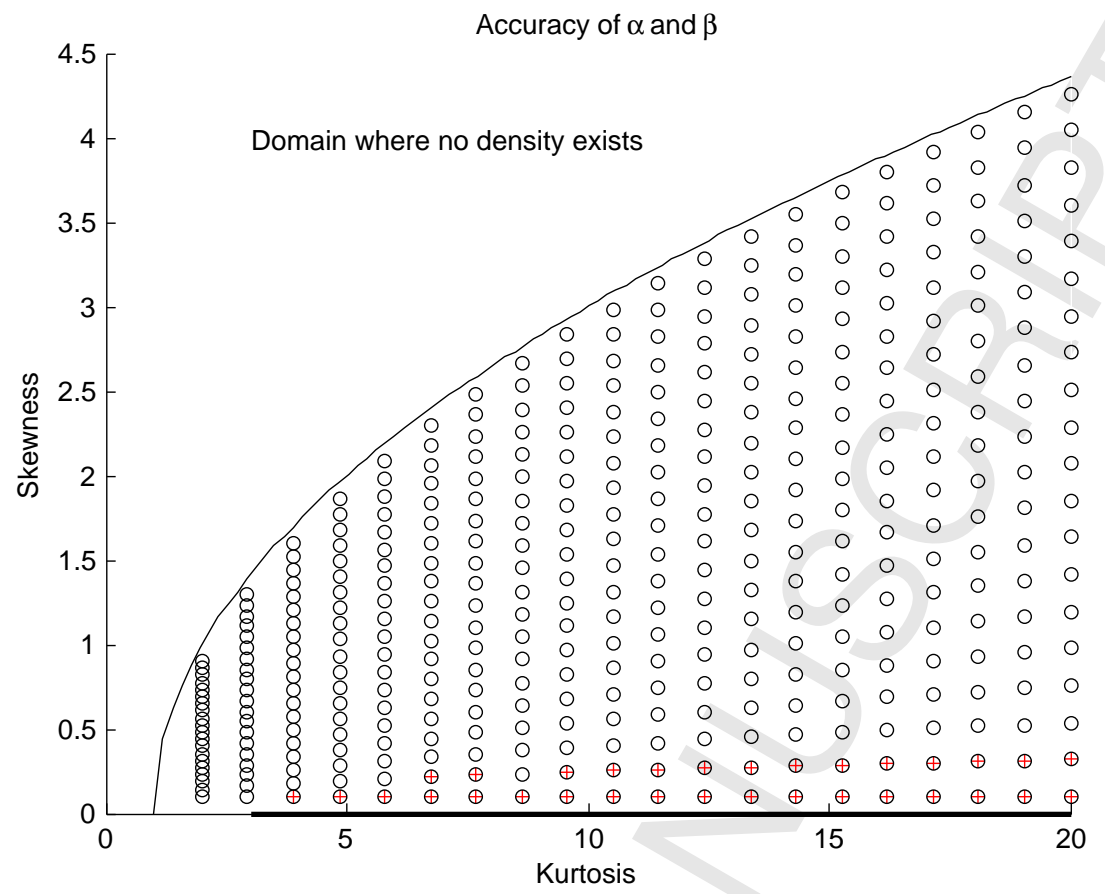

FiguRE 1. This figure represents the skewness-kurtosis domain for which a density exists (the domain is symmetric with respect to the horizontal axis). The circles represent those points for which we computed the parameters $\alpha$ and $\beta$. The symbol + represents those points for which the distance between the original skewness and kurtosis and the recomputed skewness and kurtosis (after evaluation of the $\alpha$ and $\beta$ ) is larger than $10^{-5}$. 